\title{
Near infrared surface photometry of late-type Virgo cluster galaxies ${ }^{\star}$
}

\author{
A. Boselli ${ }^{1-3}$, R.J. Tuffs ${ }^{1}$, G. Gavazzi ${ }^{4,5}$, H. Hippelein ${ }^{6}$, and D. Pierini ${ }^{4,5}$ \\ 1 MPI für Kernphysik, Postfach 103980, D-69117 Heidelberg, Germany \\ 2 DEMIRM, Observatoire de Paris, 61 Av. de l'Observatoire, 75014 Paris, France \\ 3 present address: Laboratoire d'Astronomie Spatiale, Traverse du Siphon, F-13376 Marseille Cedex 12, France \\ 4 Università degli Studi di Milano, Via Celoria 16, 20133, Milano, Italy \\ 5 Osservatorio Astronomico di Brera, Via Brera 28, 20121 Milano, Italy \\ 6 MPI für Astronomie, Königstuhl, D-69117 Heidelberg, Germany
}

Received December 22, 1995; accepted June 3, 1996

\begin{abstract}
Near Infrared ( $K^{\prime}$ band) surface photometry has been obtained for 102 (88 late-type) Virgo cluster galaxies. A subset of 20 galaxies was also imaged in the $H$ band. Magnitudes and diameters within the 21.5 and 22.0 mag $\operatorname{arcsec}^{-2}$ isophote, concentration indices and total $H$ and $K^{\prime}$ magnitudes are derived. Basic statistical properties of a complete sample of spiral galaxies spanning the range $6.3<K_{\mathrm{T}}^{\prime}<13.5$ are given. ${ }^{1}$
\end{abstract}

Key words: galaxies: fundamental parameters galaxies: photometry - infrared: galaxies - galaxies: clusters: Virgo cluster

\section{Introduction}

Samples of galaxies selected from nearby clusters are generally complete over a large range of observable properties due to their known distance. They therefore provide a natural basis for the statistical study of key physical properties such as star-formation history, gas content, metallicity, and cosmic ray production rate over a considerable dynamic range. In particular, the proximity and high galactic latitude of the Virgo cluster make it a unique laboratory for the statistical study of a complete volume-limited

Send offprint requests to: R.J. Tuffs

* based on observations taken at the Calar Alto Observatory, operated by the Max-Planck-Institut für Astronomie (Heidelberg) jointly with the Spanish National Commission for Astronomy.

1 Tables 3, 5 and 6 are only available in electronic form at the CDS via anonymous ftp to cdsarc.u-strasbg.fr (130.79.128.5) or via http://cdsweb.u-strasbg.fr/Abstract.html sample of late-type galaxies, including a full representation of quiescent and dwarf galaxies. Furthermore, compared to relaxed clusters, the effect of the cluster environment may not be so pronounced in the periphery regions of the dynamically young Virgo cluster. Most of the outlying Virgo galaxies may be falling in for the first time (e.g. Tully \& Shaya 1984; Gavazzi et al. 1991), and so may have properties more representative of field galaxies. Consequently, comparison of a deep, complete sample of Virgo cluster galaxies from the cluster-periphery with identically selected counterparts from the cluster core and (for the brighter spirals) with fully isolated galaxies should constitute a sensitive statistical measure of the effect of the cluster environment on physical processes in the galaxies.

These considerations have prompted us to embark upon a program to measure a deep, complete sample of 117 late-type Virgo cluster galaxies selected from the Virgo Cluster Catalogue of Binggeli et al. (1985) in key spectral regions to the limiting capability of available facilities. Measurements are underway in the $\mathrm{CO}$ line at $115 \mathrm{GHz}$ (Boselli et al. 1995a), in the radio continuum (Niklas et al. 1995), and in the $\mathrm{H}_{\alpha}$ line (Hippelein et al. 1996). An almost complete set of $\mathrm{HI}$ measurements from Arecibo is available (Hoffman et al. 1989 and references therein), and the sample will be measured as part of the central program of the Infrared Space Observatory using the ISOPHOT, ISOCAM and (for spiral galaxies only) LWS instruments.

In this paper we report NIR imaging observations of the majority (94) of the sample obtained at the Calar Alto observatory in 1994, 1995 and 1996. In addition, 8 other galaxies are also included. These measurements provide detailed information on the distribution of the bulk of the stars which dominate the mass in galaxy disks, rather than the younger stars which contribute more to the visible (Aaronson et al. 1979; Whitmore 1984; Gavazzi 1993), and are essentially free from effects of extinction (Peletier 
\& Willner 1992; Boselli \& Gavazzi 1994). Apart from aiding the statistical interpretation of data in other spectral ranges by acting as a mass normaliser, the NIR observations reported here will be used in conjunction with $\mathrm{H}_{\alpha}$ and blue images as a diagnostic of stellar populations within galaxy disks, and to extend earlier work (see Pierce \& Tully 1988; Peletier \& Willner 1991; Gavazzi et al. 1991) on the IR Tully-Fisher relation for Virgo cluster galaxies to fainter objects. Such aspects will be reported in future communications. Moreover the NIR observations of Virgo galaxies presented in this paper will be integrated with those of other nearby clusters (including A262, Cancer, A1367 and Coma) obtained by Gavazzi et al. (1996a,b) to extend the study of the NIR properties of disk galaxies to a wider luminosity range. The scope of the present paper is to describe the sample selection, observations, and data reduction (Sect. 2) and to present the results in the form of images and derived aperture photometry (Sect. 3). Since observations of almost all the spiral galaxies in the sample have been completed in $K^{\prime}$, the luminosity function of spirals in $K^{\prime}$ is given and briefly discussed in Sect. 4 together with some other simply derived quantities.

\section{Sample definition, observations and data reduction}

The sample was selected from the Virgo Cluster Catalogue (VCC) of Binggeli et al. (1985, 1993), which is complete to $B_{\mathrm{T}}=18$, by applying the following criteria:

$-B_{\mathrm{T}}<18$.

- Hubble type later than S0.

- Classified as cluster member by Binggeli et al. (1985, 1993).

Limitations on telescope time dictated that not all the 207 VCC galaxies satisfying these criteria could be observed. Therefore, a further criterion based on projected position was introduced, as sketched in Fig. 1.

The sky areas from which galaxies were chosen define two contrasting subsamples to optimise the statistical evaluation of the cluster environment on observed properties. The cluster-core subsample is comprised of 44 galaxies with projected positions within 2 degrees of $\mathrm{M} 87$, essentially from within the X-ray emitting "atmosphere" of M 87 (Boehringer et al. 1994). The cluster-periphery subsample is comprised of 73 galaxies with angular separations of greater than 4 degrees from the position of maximum projected galaxy density given by Sandage et al. (1985), but excluding galaxies within 1.5 degrees of the M49 sub-cluster. To limit the spread of distances within the sample, galaxies towards the $\mathrm{M}$ and $\mathrm{W}$ clouds, and in the Southern extension $\left(\delta<5^{\circ}\right)$ were also excluded.

The resulting sample of 117 galaxies is complete to $B_{\mathrm{T}}=18$, and both the cluster-periphery and -core subsamples span the range $10<B_{\mathrm{T}}<18$. Both subsamples are approximately equally divided between giant spirals

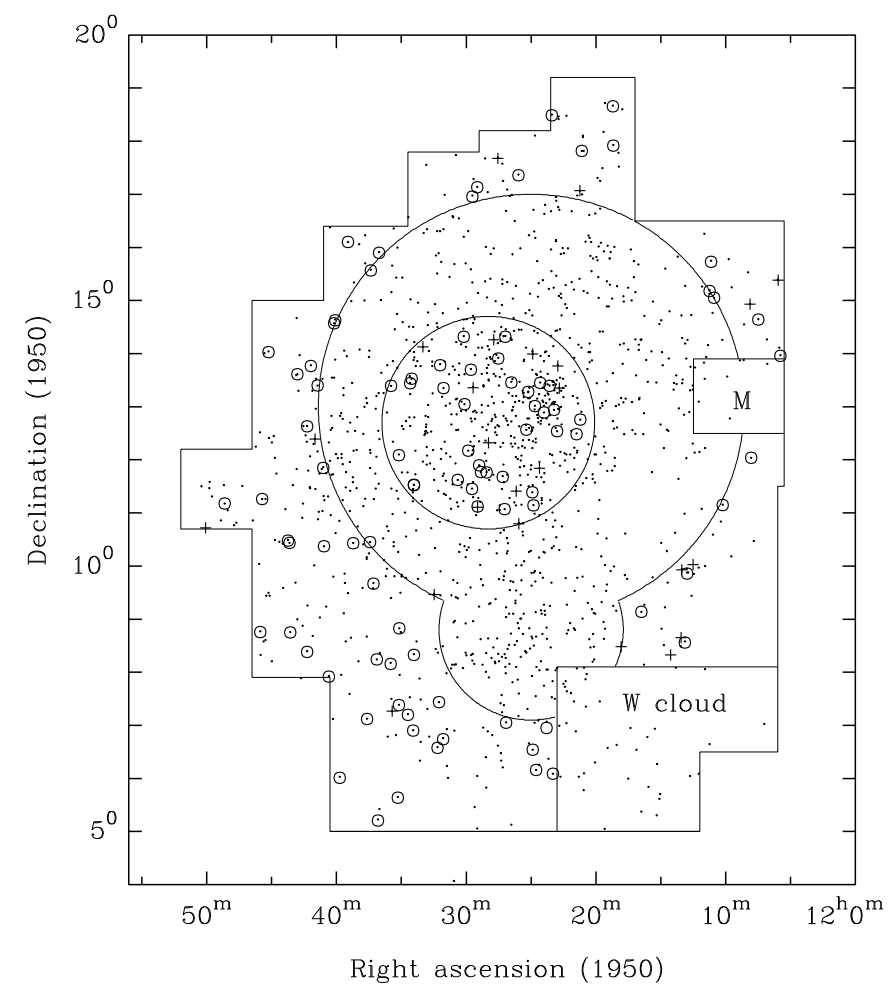

Fig. 1. Plot of all VCC galaxies classified as members by Binggeli et al. (1985) taken from Fig. 1 of Sandage et al. (1985). The subsample of late-type galaxies described in Sect. 2 are marked either with $\odot$ (objects observed in this work) or with + (objects in the sample still to be observed). The dots indicated all remaining VCC galaxies, irrespective of type and membership. The 2.0 degree radius circle centred on M 87 contains the cluster-core subsample. The inner boundary of the cluster periphery subsample has a radius of 4 degrees about the position of maximum projected galaxy density

on the one hand and dwarf and irregular galaxies on the other. The distribution over Hubble type is summarised in Table 1.

Table 1. Distribution of sample over Hubble type for the cluster-periphery and cluster-core subsamples

\begin{tabular}{lccccc}
\hline & S0/a-Sab & Sb-Sc & Scd-Sm & Im & BCD \\
\hline periphery & 8 & 15 & 19 & 23 & 8 \\
core & 13 & 10 & 9 & 10 & 2 \\
Total & 21 & 25 & 28 & 33 & 10 \\
\hline
\end{tabular}

In this paper we report NIR observations of 94 of the 117 selected galaxies. This subsample, which is complete to $B \leq 16.0 \mathrm{mag}$, includes all the $\mathrm{SO} / \mathrm{a}$ (6 objects), 62 of the 63 spirals and 25 of the $43 \mathrm{Im}$-BCDs. 


\subsection{Observations}

The observations were carried out in February 1994 and 1995 with the Calar Alto 2.2-m telescope and in February 1996 with the Calar Alto 3.5-m telescope. The Cassegrain focus of both telescopes was equipped with the MAGIC $256 \times 256$ pixel NICMOS3 infrared array (Herbst et al. 1993). In order to observe galaxies with large apparent sizes, the optical configuration of the detector at the 2.2-m telescope was chosen to give the largest possible field of view, i.e. $6.8 \times 6.8 \mathrm{arcmin}^{2}$, with a pixel size of 1.61 arcsec. For one night in 1995 (February 15) we used an optical configuration with a pixel size of 0.64 arcsec and a field of view of $2.7 \times 2.7 \mathrm{arcmin}^{2}$.

Fifteen galaxies have been observed at the 3.5-m telescope: 4 of them were objects observed in non-photometric conditions at the 2.2-m telescope for which a calibration was necessary, 2 were low surface brightness galaxies already observed at the $2.2-\mathrm{m}$ telescope but with a poor signal to noise, and 9 were new measurements. The optical configuration of the detector at the 3.5-m telescope gave a pixel size of 0.81 arcsec and a field of view of $3.4 \times 3.4 \operatorname{arcmin}^{2}$. To improve the signal to noise, the images of the galaxies observed with both telescopes were scaled to the same resolution and combined. Out of the 17 scheduled nights, 7 were useful, as specified in the log book of the observations given in Table 2 . All observations were obtained with a seeing of typically $1-2$ arcsec.

Table 2. The logbook of the observations

\begin{tabular}{lllll}
\hline year & tel & day & quality & pixel size \\
\hline 1994 & 2.2 & Feb 24 & Photometric & 1.61 \\
1994 & 2.2 & Feb 25 & Photometric & 1.61 \\
1995 & 2.2 & Feb 10 & Photometric & 1.61 \\
1995 & 2.2 & Feb 12 & Partly Photometric & 1.61 \\
1995 & 2.2 & Feb 13 & Partly Photometric & 1.61 \\
1995 & 2.2 & Feb 15 & Partly Photometric & 0.64 \\
1996 & 3.5 & Feb 12 & Partly Photometric & 0.81 \\
\hline
\end{tabular}

Table 3 (only available in electronic form) lists the relevant parameters of the galaxies observed in this work. The Table is arranged in order of increasing VCC name, as follows:

Column 1: VCC denomination (Binggeli et al. 1985). Galaxies marked * are not included in the sample as defined in Sect. 2.

Columns 2, 3: NGC and UGC denominations (Nilson 1973).

Columns 4, 5: adopted (1950) celestial coordinates.

Column 6: morphological type, from Binggeli et al. (1985). Columns 7, 8: major and minor optical $B$ band diameters, in arcminutes, from Binggeli et al. (1985) $\left(D_{25.5}(B)\right)$.
These diameters are isophotal diameters at $25.5 \mathrm{mag}$ $\operatorname{arcsec}^{-2}$.

Column 9: photographic magnitude, from Binggeli et al. (1985).

Columns 10 and 11: total $H$ and $K^{\prime}$ magnitudes obtained by interpolating the present photometric measurements to the optical diameter along circular apertures as outlined in Gavazzi \& Boselli (1996). The $H$ magnitudes of galaxies not observed in this work were determined using the aperture photometry available in the literature, following Gavazzi \& Boselli (1996). These magnitudes are uncorrected for internal extinction, extinction due to our Galaxy and $K$ dimming.

Obtaining a satisfactory background subtraction is the main difficulty of IR observations. At $1.65 \mu \mathrm{m}$ and $2.1 \mu \mathrm{m}$ the sky brightness at Calar Alto was typically 13.8 and $13.1 \mathrm{mag} \operatorname{arcsec}^{-2}$ respectively. Because of the predominance of faint extended sources in the sample, the principal waveband was chosen to be $K^{\prime}$ rather than $H$, as the sky was somewhat more stable in the former band, allowing flatter fields to be obtained. The $K^{\prime}$ band (Wainscoat \& Cowie 1992) was preferred to $K$ to reduce the effects of thermal emission from the sky, and from the telescope. At $K^{\prime}$ the sky brightness varied over the time scale of an observation by typically $1 \%$ in photometric conditions. The sky variability ranged up to $5 \%$ in the worst conditions encountered. Reaching a brightness limit $8-9$ mag $\operatorname{arcsec}^{-2}$ fainter than the sky requires a careful subtraction of the sky, necessitating mosaicing techniques (which mimic the function of the chopping secondary used with aperture photometry IR measurements).

Three types of mosaic maps, obtained by programming the telescope pointing along different patterns, were used. Galaxies with optical diameter larger than half of the size of the field of view of the array were observed using a mosaic in which $50 \%$ of the time is devoted to the target of interest and $50 \%$ to the surrounding sky ("A" mosaic, Fig. 2a). This pattern was obtained alternating 8 fields centred on the target with 8 observations of the sky chosen along a circular path around the galaxy (off-set by a field of view from the centre). The 8 on-target fields were dithered by 10 arcsec in order to help the elimination of bad pixels.

Galaxies with optical diameter smaller than half of the size of the field of view of the array were observed with a mosaic consisting of 9 pointings along a circular path and displaced from one-another by 2 arcmin such that the target galaxy is always in the field ("B" mosaic; Fig. 2b). To avoid saturation each pointing was split into several short elementary integrations of $2-4 \mathrm{~s}$ which were added by the on-line MAGIC software. There were three galaxies with angular sizes larger than the dimension of the detector; these were mapped using mosaics expressly prepared according to the shape and orientation of the galaxy in the sky in order to cover the entire surface of the target. In order to get a higher signal-to-noise the mosaics were 

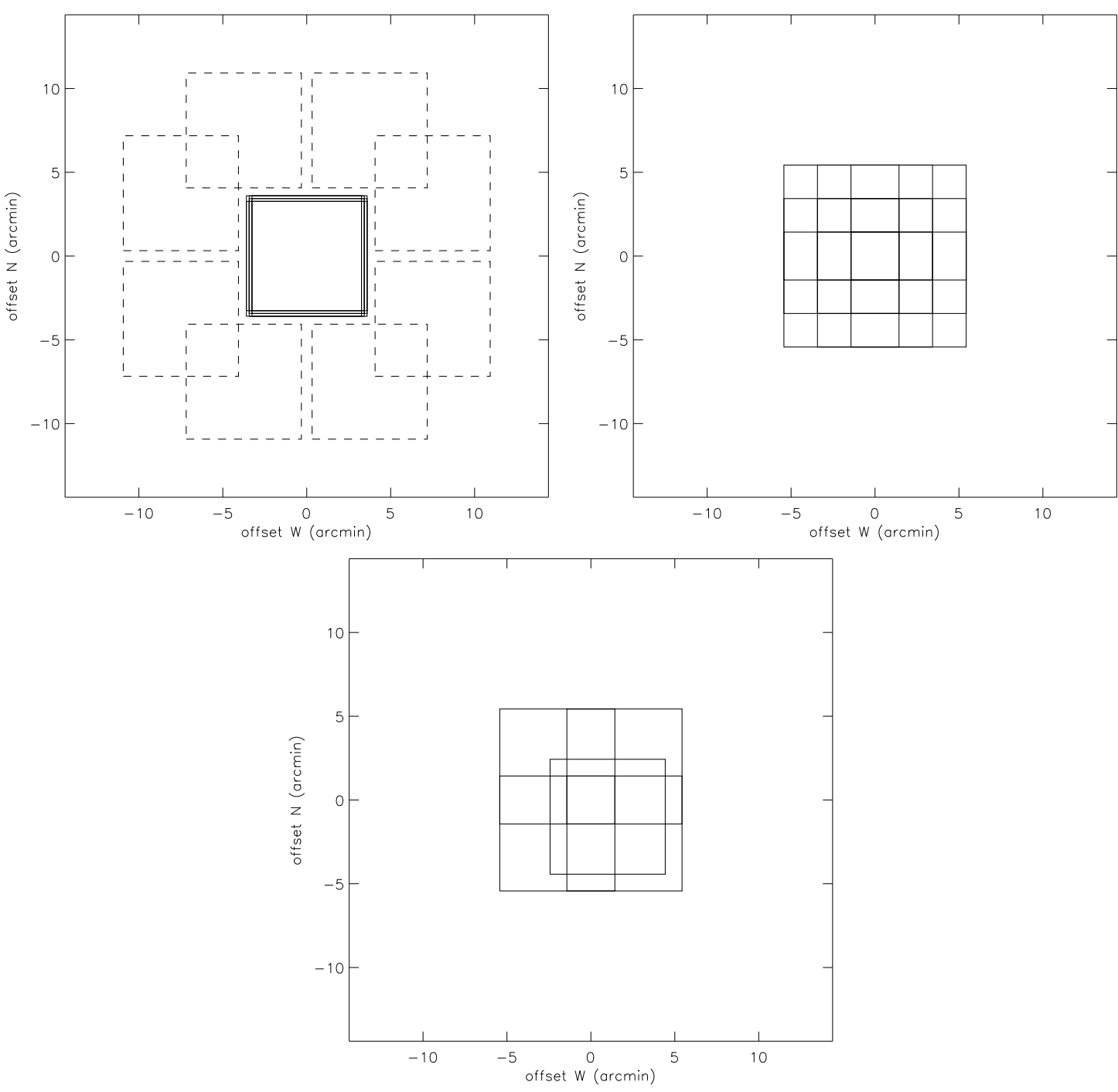

Fig. 2. Typical mosaic patterns used during the observations with the 2.2-m telescope and the 1.61 arcsec/pixel scale: a) "A" mosaic, b) "B" mosaic and c) "C" mosaic. The figures show the outline of the detector array at the positions observed. The dashed lines in mosaic "A" delineate the sky reference positions

repeated for low surface brightness galaxies.

Calibration stars were observed with a third mosaic ("C", Fig. 2c). This is composed of 5 pointings, starting with the target star near to the centre of the array followed by pointings in each of the 4 quadrants of the array.

\subsection{Photometric calibration}

The observations were calibrated and the fluxes transformed into the $H$ and $K^{\prime}$ photometric system using the standard stars listed in Table 4 (Elias et al. 1982), observed hourly throughout the night. The observations of the standard stars were obtained with a defocused telescope to avoid saturation.

Only HD 40335 has a reference $K^{\prime}$ mag (Wainscoat \& Cowie 1992). For the others stars, having almost null spectral slopes, $K^{\prime}$ was determined using the relation given by
Wainscoat \& Cowie (1992): $K^{\prime}-K=0.22(H-K)$. The typical uncertainty of the measurements in photometric periods is $0.05 \mathrm{mag}$.

Table 4. Calibration stars

\begin{tabular}{llll}
\hline name & $H$ & $K$ & $K^{\prime}$ \\
\hline HD 40335 & 6.470 & 6.450 & $6.445^{*}$ \\
HD 84800 & 7.530 & 7.530 & 7.530 \\
HD 106965 & 7.335 & 7.315 & 7.319 \\
HD 129653 & 6.940 & 6.920 & 6.924 \\
\hline
\end{tabular}

* $K^{\prime}$ value from Wainscoat \& Cowie (1992). 


\subsection{Image analysis}

The reduction of two-dimensional IR frames followed a procedure based on the IRAF data reduction package developed by NOAO and on the SAOIMAGE and PROS packages developed at the Center for Astrophysics ${ }^{2}$.

To remove the detector response, two sets of flat-field exposures were obtained on the telescope dome with (lamp-on) and without (lamp-off) illumination with a quartz lamp. The response of the detector is then contained in the normalized frame $F F=$ [(lamp-on) (lamp-off)] / 〈(lamp-on) - (lamp-off) $\rangle$ (per pixel).

Specific reduction strategies were used for the various mosaics, according to the stability of the sky during the observations. When the sky was stable to within a few percent during the observation of a galaxy (the large majority of the observations), the $8 \mathrm{SKY}$ exposures $\left(\mathrm{SKY}_{i}\right)$ were combined using a median filter to obtain $\langle\mathrm{SKY}\rangle$ for type "A" mosaics. For type "B" mosaics $\langle\mathrm{SKY}\rangle$ was obtained by combining the 9 frames containing target $+\mathrm{SKY}$ with a median filter.

The mean counts $\left\langle c_{\mathrm{T}}\right\rangle_{i}$ and $\left\langle c_{\mathrm{sky}}\right\rangle$ were respectively determined for the $i^{\text {th }}$ target observation and the median sky. Individual "normalized" $\mathrm{SKY}_{i}$ frames were then produced such that $\mathrm{SKY}_{i}=\langle\mathrm{SKY}\rangle \times\left\langle c_{\mathrm{T}}\right\rangle_{i} /\left\langle c_{\text {sky }}\right\rangle$. This removed the time variations of the sky level, but, due to the source emission, introduced an (additive) offset; this was subsequently removed (see below). Occasionally, when the average response of the detector to the sky changed by about $5 \%$ during an observation, significant temporal variations in the spatial response of the detector to the sky became discernable. Under these circumstances, only the three sky frames closest in time to each target frame were used to determine the sky. After sky removal, each target frame $\left(T_{i}\right)$ was processed to obtain a flat-field, sky subtracted, corrected frame: $T_{i, \text { corr }}=\left[T_{i}-\mathrm{SKY}_{i}\right] / F F$.

Sky-subtracted and flat-fielded frames were then registered using field stars and combined together with a median filter. This provided a satisfactory removal of the bad pixels in the final combined image. Tests on the data showed that the photometry obtained from this use of a median filter was identical to that obtained with averaging techniques.

Star-subtracted frames were produced by a manual "editing" of the contribution from pointlike sources which are clearly not associated with the target galaxies.

The residual sky background and its rms noise $(\sigma)$ (in the individual pixels) were determined in each star-subtracted

2 IRAF is the Image Analysis and Reduction Facility made available to the astronomical community by the National Optical Astronomy Observatories, which are operated by AURA, Inc., under contract with the U.S. National Science Foundation. STSDAS is distributed by the Space Telescope Science Institute, which is operated by the Association of Universities for Research in Astronomy (AURA), Inc., under NASA contract NAS5-26555. frame in concentric object-free annuli around the objects of interest.

We checked the quality of the final images on large and small scales. On small scales the measured noise was always consistent with the expected statistical fluctuations in the photon count from the sky background accumulated over the total integration time. Over scales comparable with the sizes of the galaxies, the images are limited by imperfections in the sky subtraction. Typically, the deviation from flatness from this effect is $0.05 \%$ or better for photometric conditions, and $0.08 \%$ for non-photometric conditions. Although it has an amplitude of only about $10 \%$ of the statistical fluctuations on individual pixels, this deviation is the limiting factor in the virtual aperture photometry.

\section{Results}

\subsection{The virtual aperture photometry and the second order photometric calibration}

The present data were compared with aperture photometry available in the literature by integrating the counts in concentric circular rings around the galaxy centres to provide curves of growth up to the diameter of the reference photometry. This operation was made without subtracting stars from the frames since, unless otherwise specified, the aperture photometry is generally not corrected for stellar contamination. Galaxies observed at the 2.2-m telescope in non photometric conditions and with no aperture photometry available in the literature were re-observed at the $3.5 \mathrm{~m}$ telescope. The growth curve was used to derive the photometric zero point for galaxies observed in non photometric periods (as indicated in Table 5), and provided a general check of the intrinsic photometric accuracy of the current work. The virtual photometry measurements obtained in this work are consistent in the average with the aperture photometry available in the literature (88 measurements): $H_{\text {this work }}-H_{\text {literature }}=-0.036 \pm 0.140 \mathrm{mag}$ and $K_{\text {this work }}^{\prime}-K_{\text {literature }}^{\prime}=-0.024 \pm 0.151 \mathrm{mag}$. The most discrepant measurements are those at small apertures $(5-15$ arcsec) due to a combination of seeing effects and galaxy centering, which might be quite inaccurate in the objects with no bright nuclei such as dwarf irregular galaxies.

We estimate that the overall photometric accuracy of our data, including systematic errors on the zero point determination is $\leq 0.1 \mathrm{mag}$.

To facilitate comparison with optical data available in the literature, integrated magnitudes were determined in circular apertures from the star-subtracted frames to the diameter of the $25.5 \mathrm{mag} \operatorname{arcsec}^{-2}$ blue isophote as described and tabulated in Table 3 . 


\subsection{Photometry along elliptical annuli}

Using the star-subtracted frames, the surface brightness profiles were re-determined by averaging the brightness distribution in concentric elliptical annuli of fixed centre, position angle and ellipticity. The ellipses were fitted by eye to the $K^{\prime}$ band $21 \mathrm{mag} \operatorname{arcsec}^{-2}$ isophotes of the galaxy under study (for some low-surface brightness galaxies, a weaker isophote had to be used: the values are given in parentheses in Table 5; see Sect. 4). This simple technique was preferred to more sophisticated procedures to be as consistent as possible with the method we used in optical CCD studies (see Gavazzi et al. 1995a and references therein). Given the irregular shape of Im and BCD galaxies, elliptical profiles give just a crude representation of their real profiles (see Sect. 4). Starting from an inner ellipse of size comparable with the seeing disk, a set of annuli, increasing in major axis by fixed amounts was drawn. In each annulus the total number of counts, pixels and associated statistical uncertainties were computed following Gavazzi et al. (1994).

The isophotal major radii $\left(a_{21.5}\right)$ in the $H$ and $K^{\prime}$ bands were determined from the azimuthally integrated profiles as the radii at which the surface brightness reaches $21.5 \mathrm{mag} \operatorname{arcsec}^{-2}$. The values of $a_{21.5}$ given in this work are not corrected for galaxy inclination.

Magnitudes were derived by integrating the elliptical light profiles up to the radius corresponding to the $21.5 \mathrm{mag} \operatorname{arcsec}^{-2}$ isophote $\left(H_{21.5}\right.$ and $\left.K_{21.5}^{\prime}\right)$. Since in some dwarf galaxies $21.5 \mathrm{mag} \operatorname{arcsec}^{-2}$ is too close to the peak brightness to measure a meaningful diameter, we also give $a_{22.0}$ and $K_{22.0}^{\prime}$. These entries, which are generally less accurate than $a_{21.5}$ and $K_{21.5}^{\prime}$ because of the higher noise in the data at the $22.0 \mathrm{mag} \operatorname{arcsec}^{-2}$ isophote, can nevertheless be fairly well determined in the low surface brightness galaxies where longer integration times were used. These magnitudes are observed quantities and are not corrected for extinction. The photometric parameters determined along elliptical rings are summarized in Table 5 (only available in electronic form), as follows:

Column 1: VCC name.

Column 2: adopted filter.

Column 3: total integration time per position (in sec); this corresponds to the product of the exposure time of the elementary integration (generally $2-4 \mathrm{~s}$ ) $\times$ the number of (added) elementary integrations.

Column 4: number of frames per galaxy (combined with a median filter).

Column 5: adopted mosaic, as defined in Sect. 2.2. Some galaxies (marked $\times 1$ ) were serendipitously observed in the sky frames of other targets. S indicates large galaxies where an ad hoc designed mosaic was used.

Column 6: pixel size, in arcseconds: these are 0.64 and $1.61 \mathrm{arcsec} / \mathrm{pixel}$ for the $2.2 \mathrm{-m}$ telescope and $0.81 \mathrm{arc}-$ $\mathrm{sec} /$ pixel for the 3.5-m telescope.

Column 7: photometric quality: galaxies marked "*" were observed in non-photometric periods; diameters and magnitudes are not determined; for galaxies marked " $r$ " the zero-point was determined using reference aperture photometry.

Column 8: position angle of the galaxy major axis (measured counterclockwise from N).

Column 9: ellipticity $(1-b / a)$.

Column 10: observed major $\left(a_{21.5}\right)$ radius (in arcsec) determined at the $21.5^{\text {th }}$ magnitude isophote.

Column 11: observed magnitude $\left(m_{21.5}\right)$ integrated within the $21.5 \mathrm{mag} \operatorname{arcsec}^{-2}$ elliptical isophote.

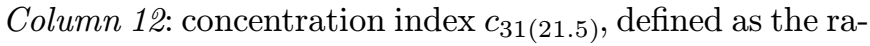
tio between the radii that contain $75 \%$ and $25 \%$ of $m_{21.5}$. Column 13: observed major $\left(a_{22.0}\right)$ radius (in arcsec) determined at the $22.0^{\text {th }}$ magnitude isophote.

Column 14: observed magnitude $\left(m_{22.0}\right)$ integrated within the $22.0 \mathrm{mag} \operatorname{arcsec}^{-2}$ elliptical isophote.

Column 15: concentration index $c_{31(22)}$, defined as the ratio between the radii that contain $75 \%$ and $25 \%$ of $m_{22.0}$.

For galaxies observed at both the 2.2-m and the $3.5-\mathrm{m}$ telescope, Table 5 reports 2 lines. The $3.5-\mathrm{m}$ telescope data refer in fact to the combined images.

Grey-level and contour representations of the $K^{\prime}$ brightness distribution of the galaxies studied are given in Fig. 3 alongside surface brightness and (where possible) colour profiles. Table 6 gives the parameters of the differential $H$ and $K^{\prime}$ band radial surface brightness profiles obtained by azimuthal integration along elliptical annuli. Table 6 (only available in electronic form) is arranged as follow:

Column 1: VCC name.

Column 2: adopted filter.

Column 3: radius (on the major axis) of the elliptical annulus, in arcseconds.

Column 4: surface brightness at the given radius.

\section{Discussion}

We have obtained NIR surface photometry of a complete volume-limited sample of late-type galaxies in the Virgo cluster. Some obvious features of this sample can be briefly discussed here. Total $B$ and $K^{\prime}$ magnitudes, taken from Table 3, will be used in the following analysis.

The relation between the optical diameters $D_{25.5}(B)$ determined at the $25.5 \mathrm{mag} \operatorname{arcsec}^{-2}$ isophote by Binggeli et al. (1985) and the $K^{\prime}$ diameters determined in this work is shown in Fig. 4. Near infrared diameters at the $22.0 \mathrm{mag} \mathrm{arcsec}^{-2}$ isophote are systematically smaller than the optical ones at the $25.5 \mathrm{mag} \operatorname{arcsec}^{-2}$ isophote.

The linear regression analysis gives the following relations:

$2 \times a_{22.0}=0.83 \times D_{25.5}(B)$ 

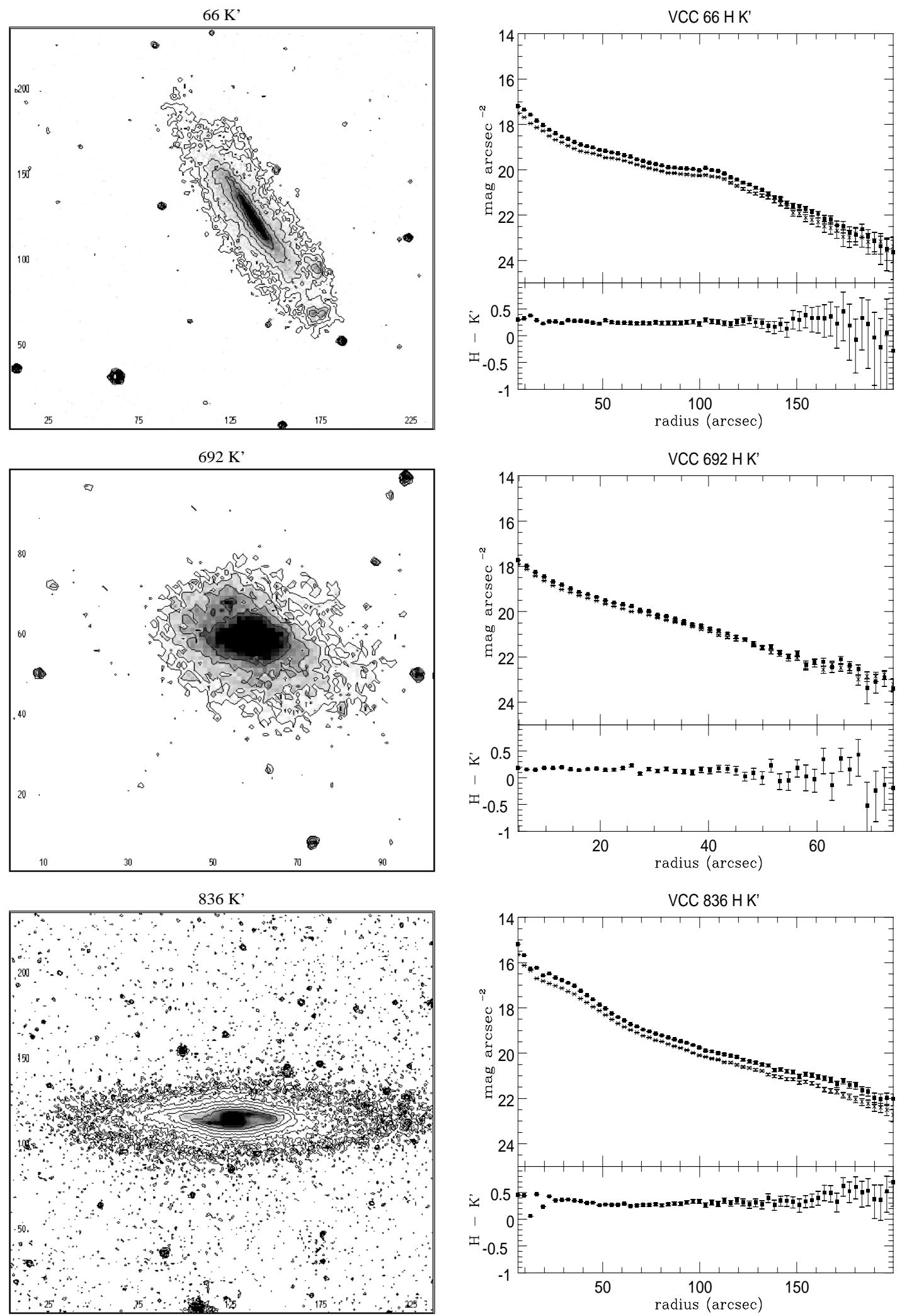

Fig. 3. a): Left hand panels: Grey-level/contour representations of the $K^{\prime}$ band brightness distributions of galaxies measured with the $2.2 \mathrm{~m}$ telescope in both $H$ and $K^{\prime}$ bands. $\mathrm{N}$ is up, E to the left. The faintest contour level shown is $20.5 \mathrm{mag}$ arcsec ${ }^{-2}$ with a step of $0.5 \mathrm{mag} \operatorname{arcsec}^{-2}$; the grey scale is arbitrary. The scale given in the image is in pixels. The angular scale of the images can be determined using the pixel size given in Table 5. Right hand panels: Differential $H$ and $K^{\prime}$ band radial surface brightness and colour profiles obtained by azimuthal integration along elliptical annuli. The horizontal scale gives the radius in arcsec as measured from the galaxy centre along the major axis of the ellipse. b): as for a) but for galaxies with only one band image available. c): as for b) but for galaxies observed with the $3.5 \mathrm{~m}$ telescope; (if both the observations are available the combined image is given). The faintest contour level shown is $21.0 \mathrm{mag} \operatorname{arcsec}^{-2}$ with a step of $0.5 \mathrm{mag} \operatorname{arcsec}^{-2}$ 

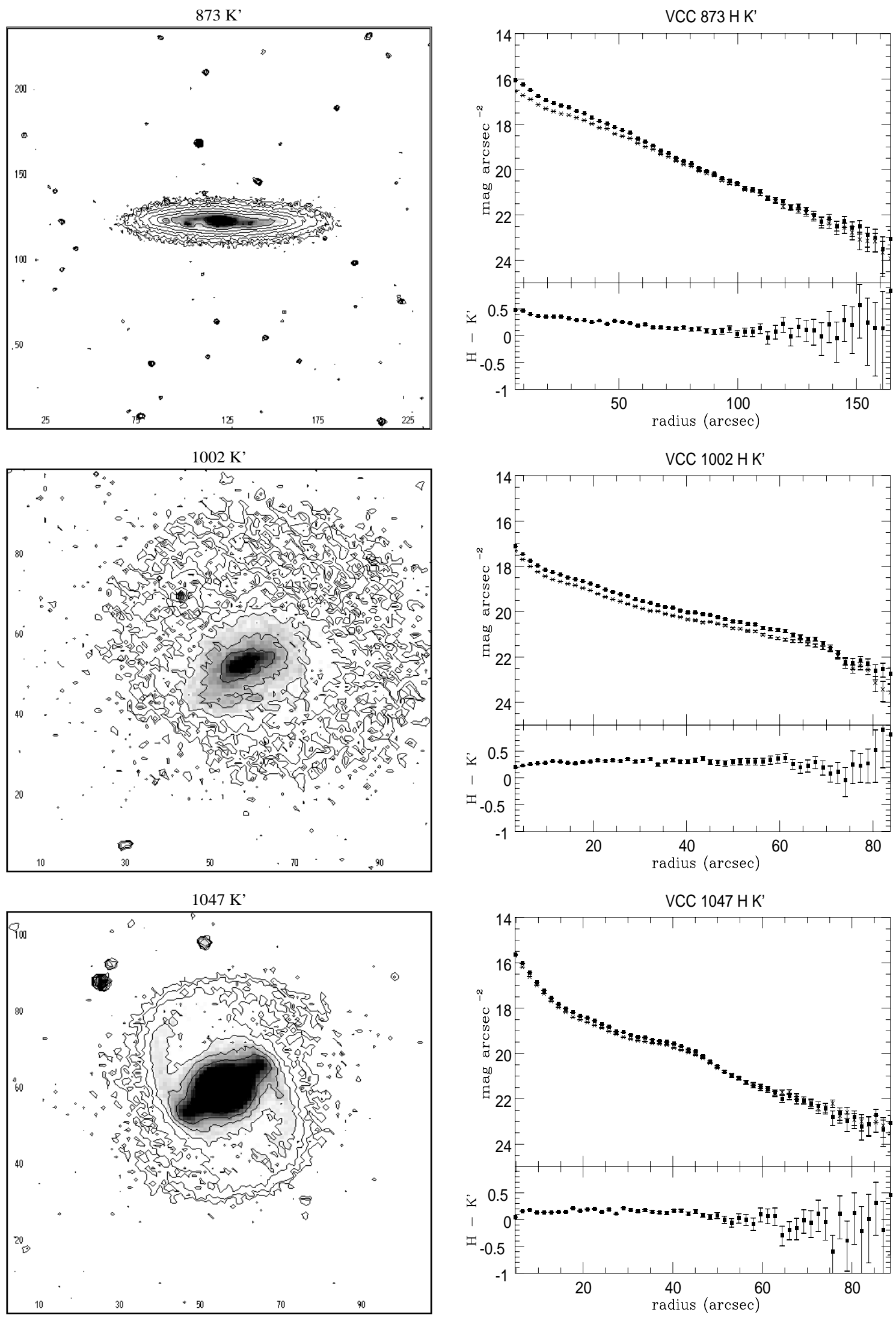

Fig. 3. a) continued 

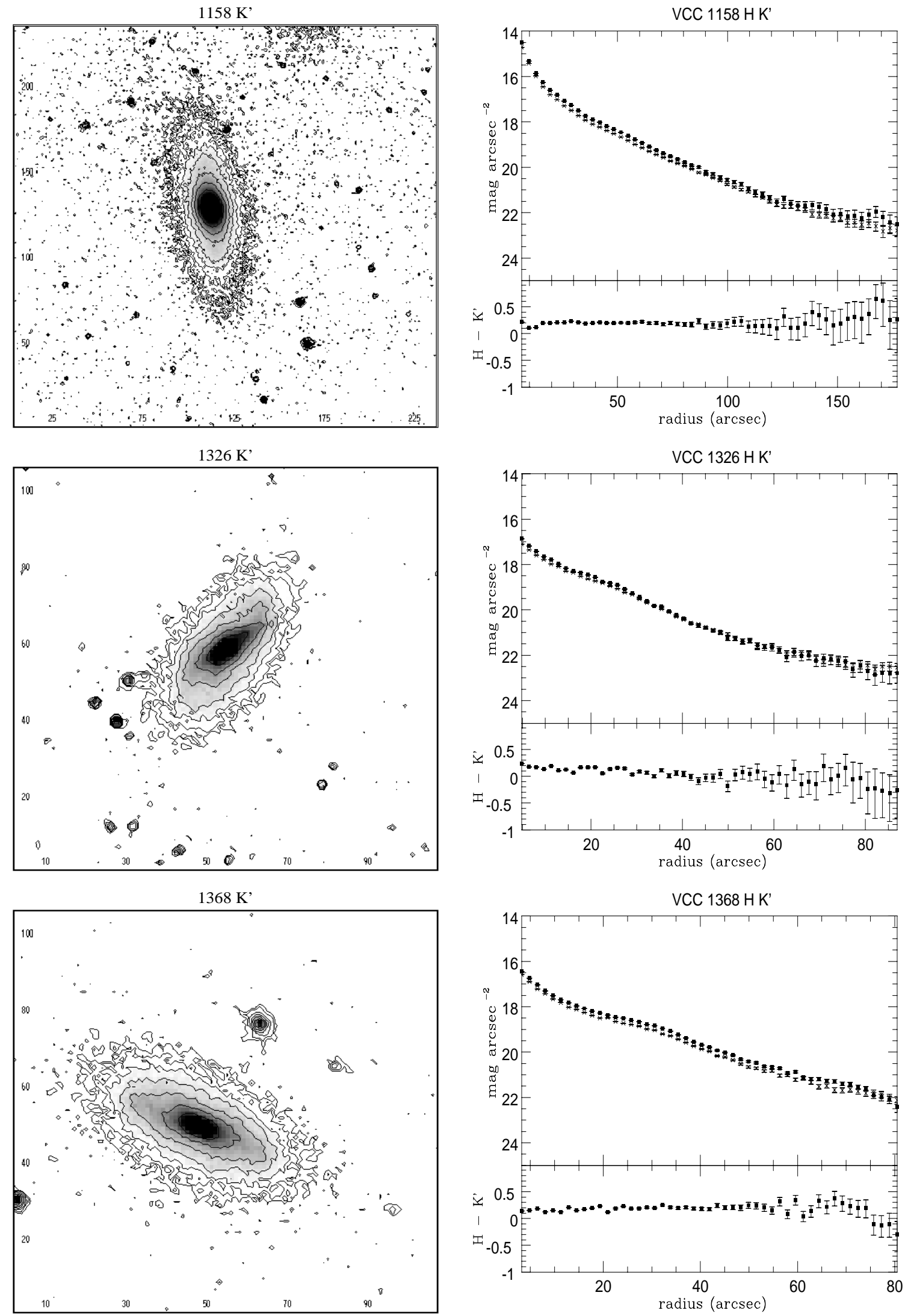

Fig. 3. a) continued 

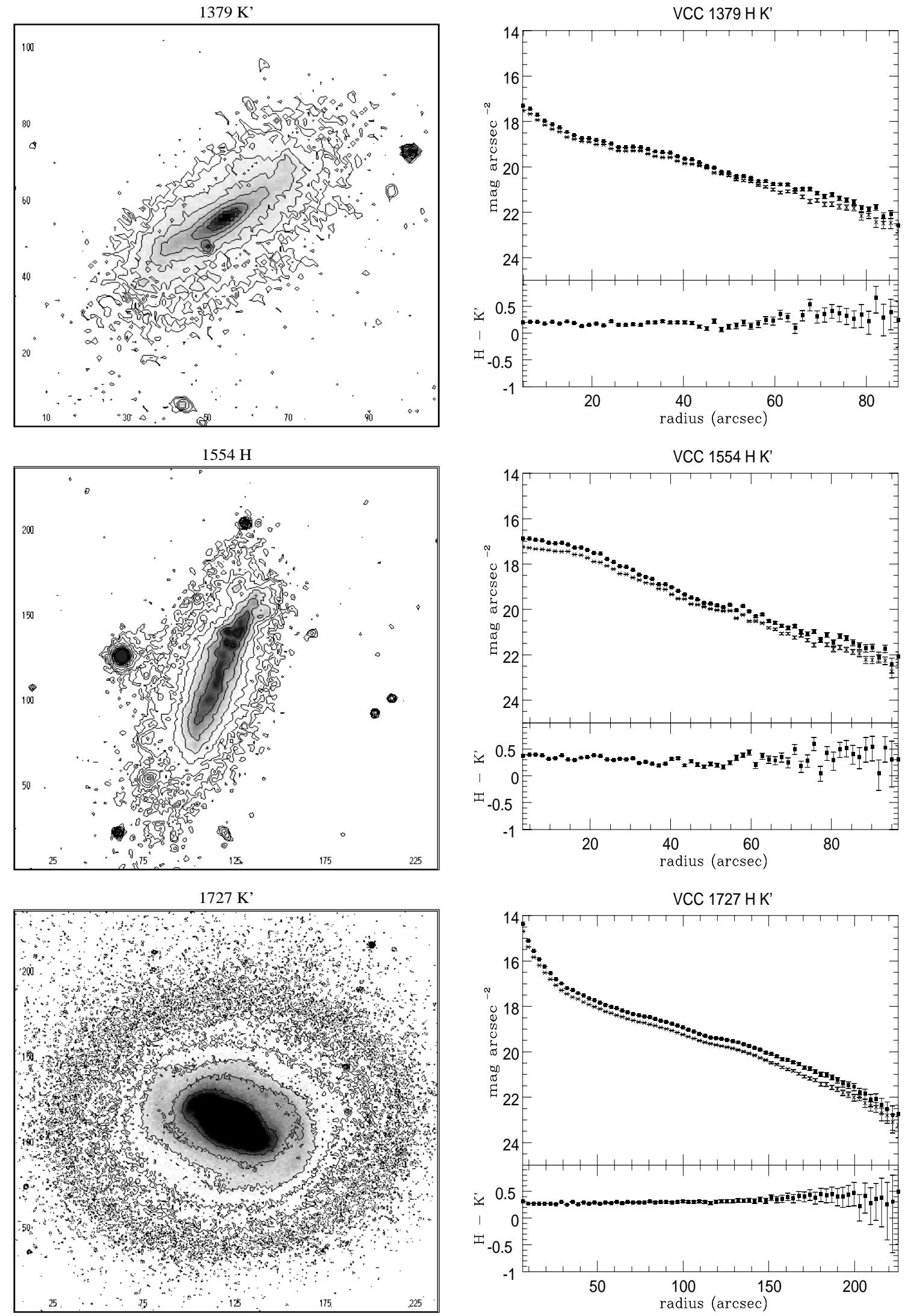

Fig. 3. a) continued 
$1811 \mathrm{~K}^{\prime}$
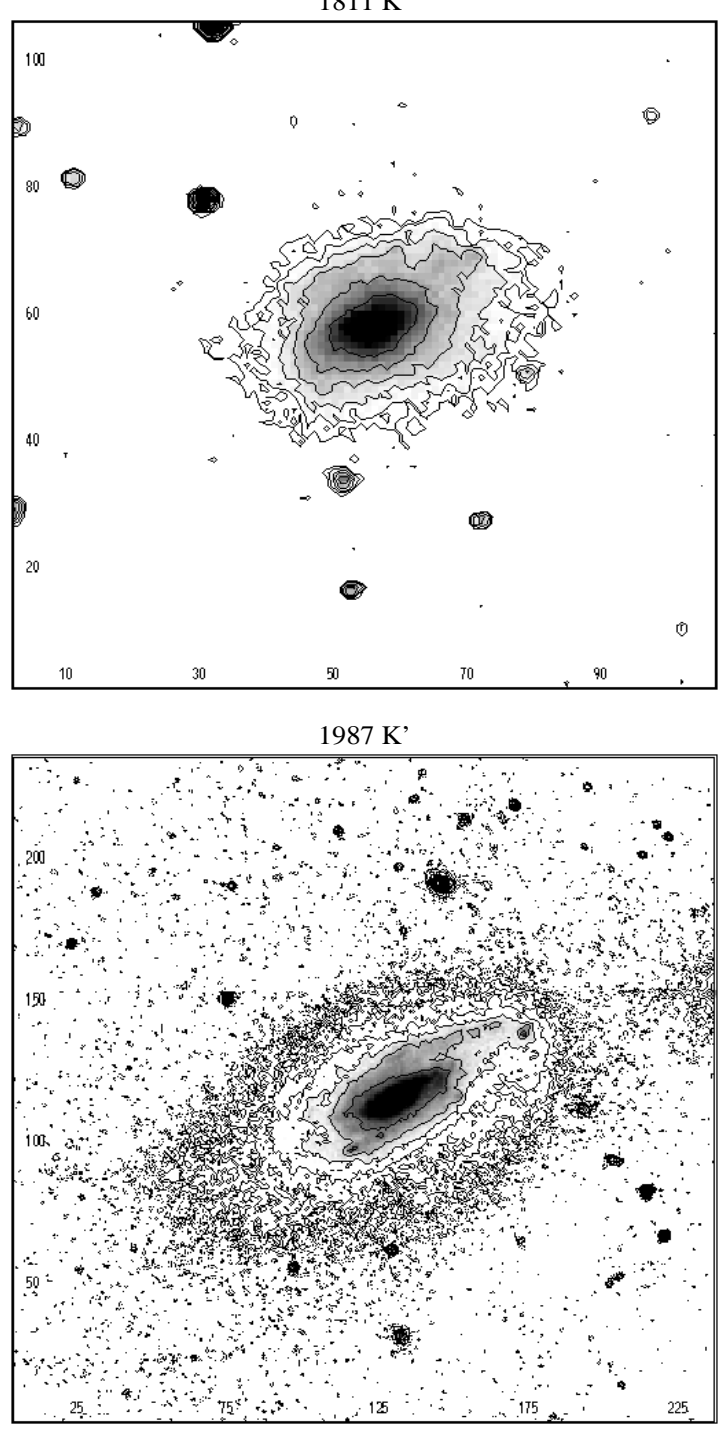
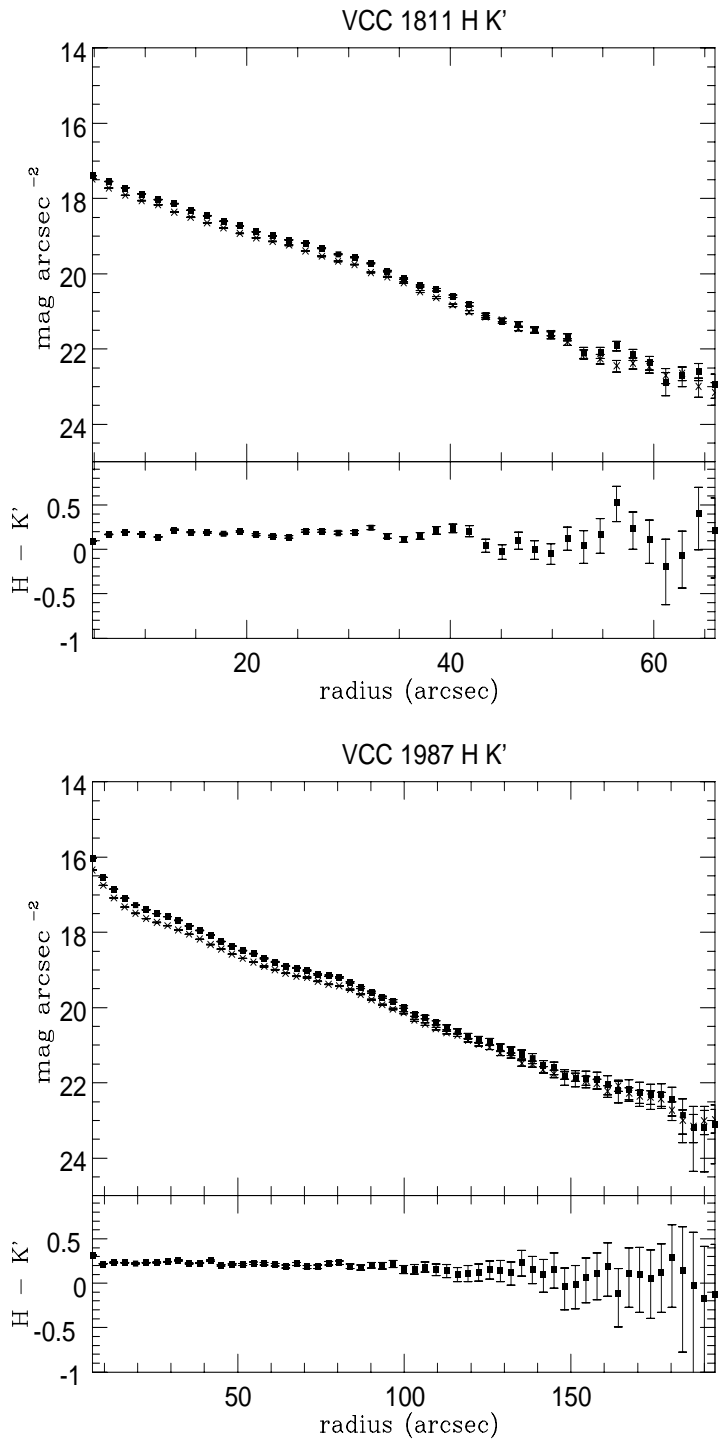

Fig. 3. a) continued 
A. Boselli et al.: NIR surface photometry of Virgo galaxies
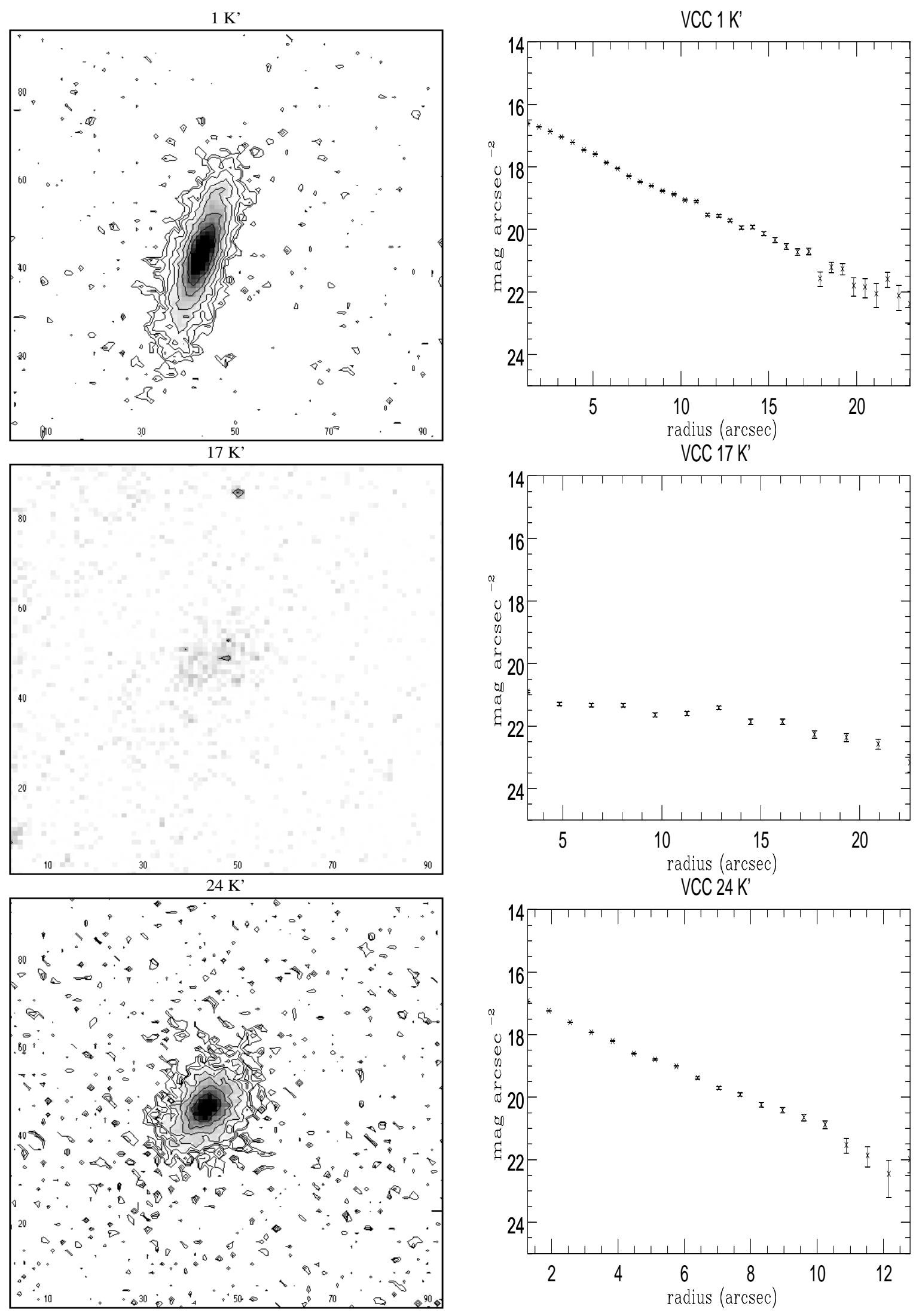

Fig. 3. b) continued 

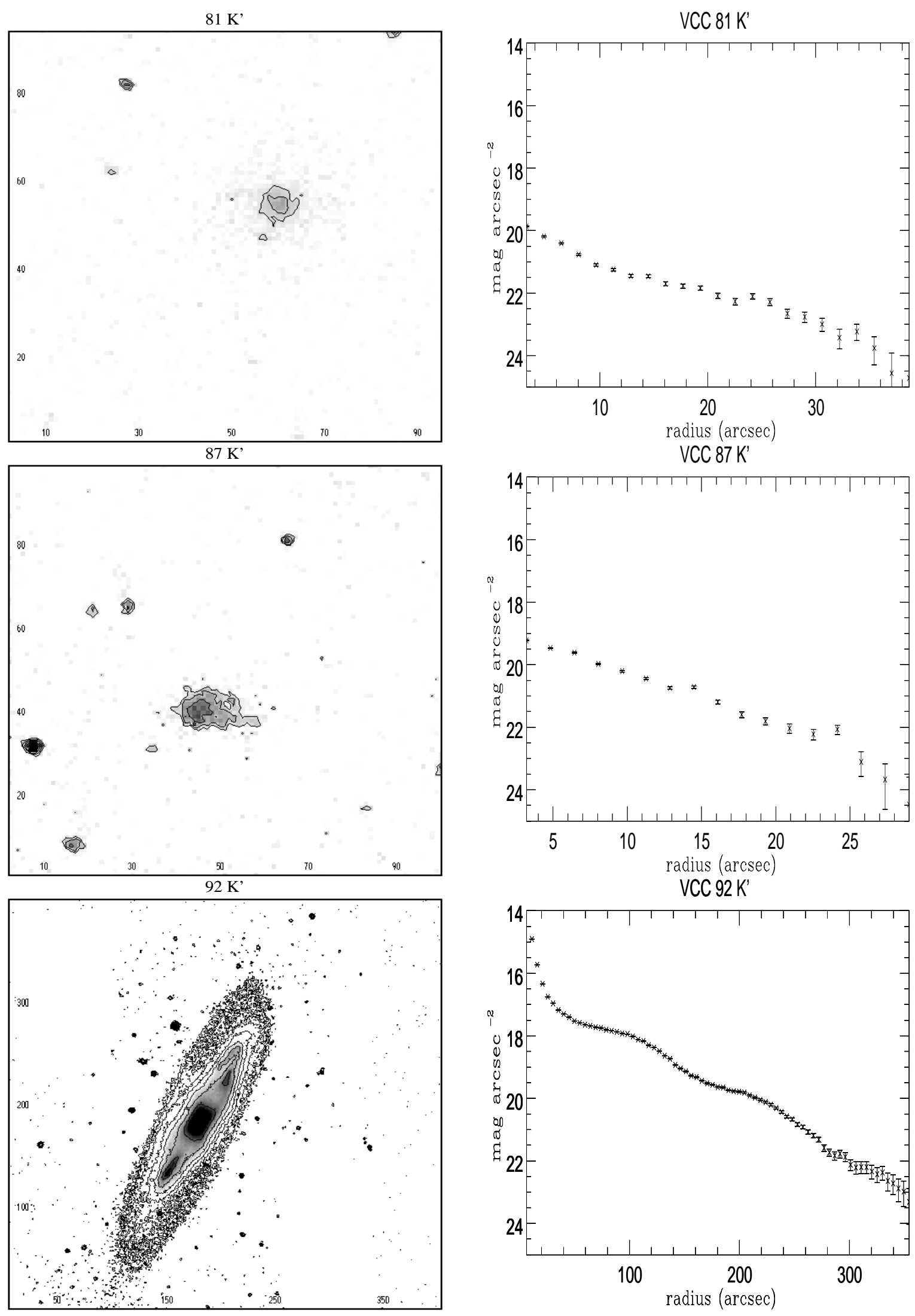

Fig. 3. b) continued 

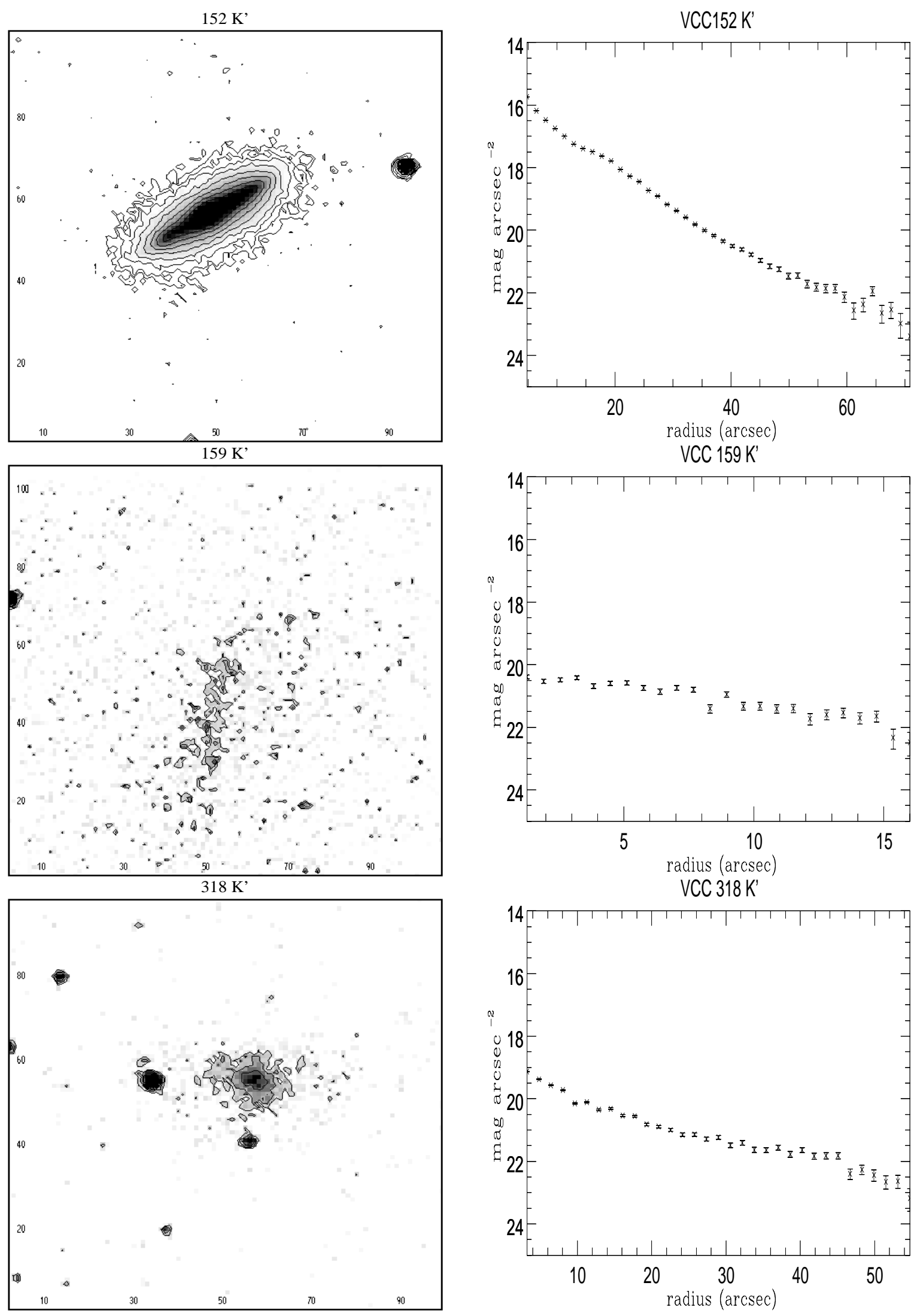

Fig. 3. b) continued 

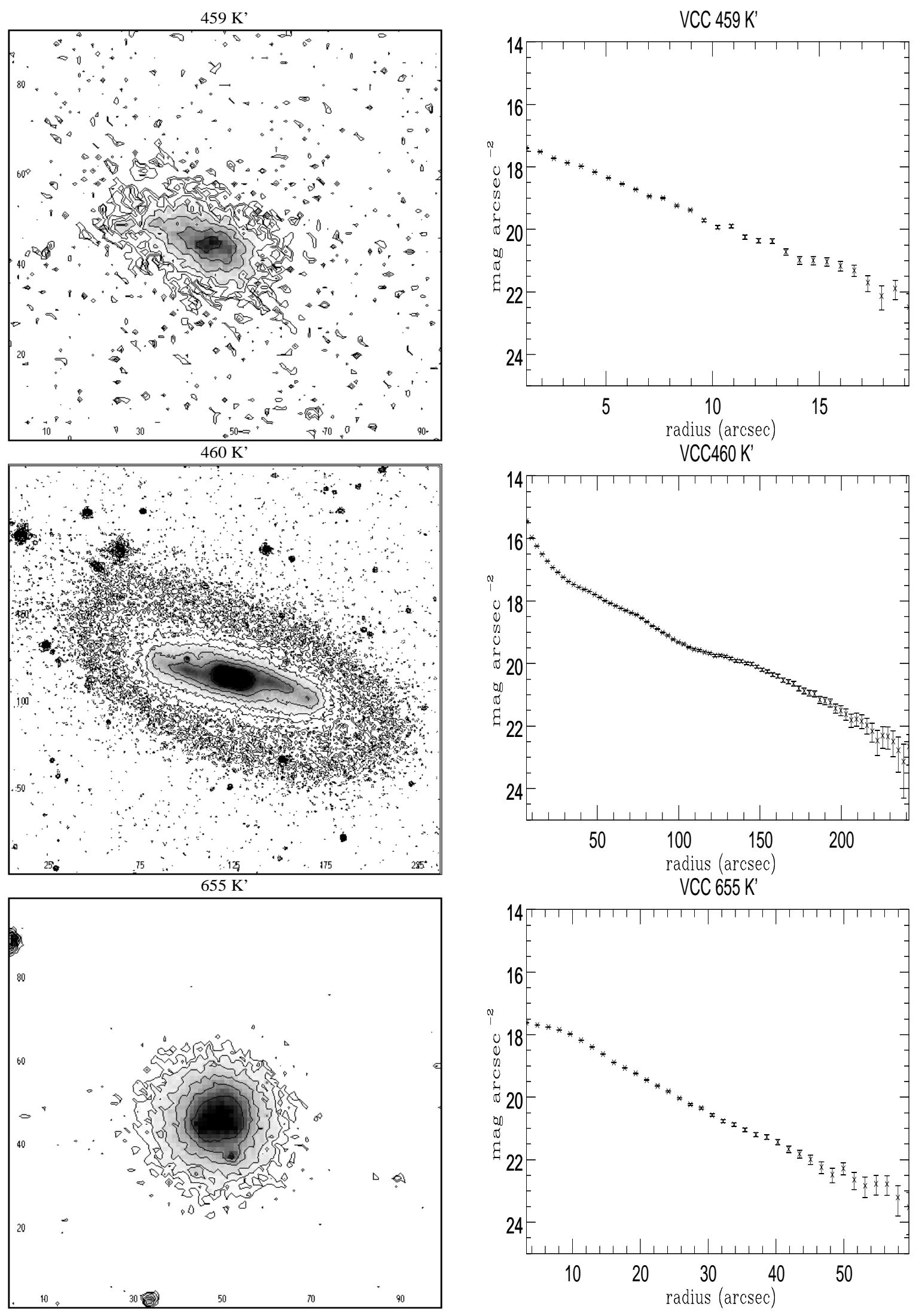

Fig. 3. b) continued 

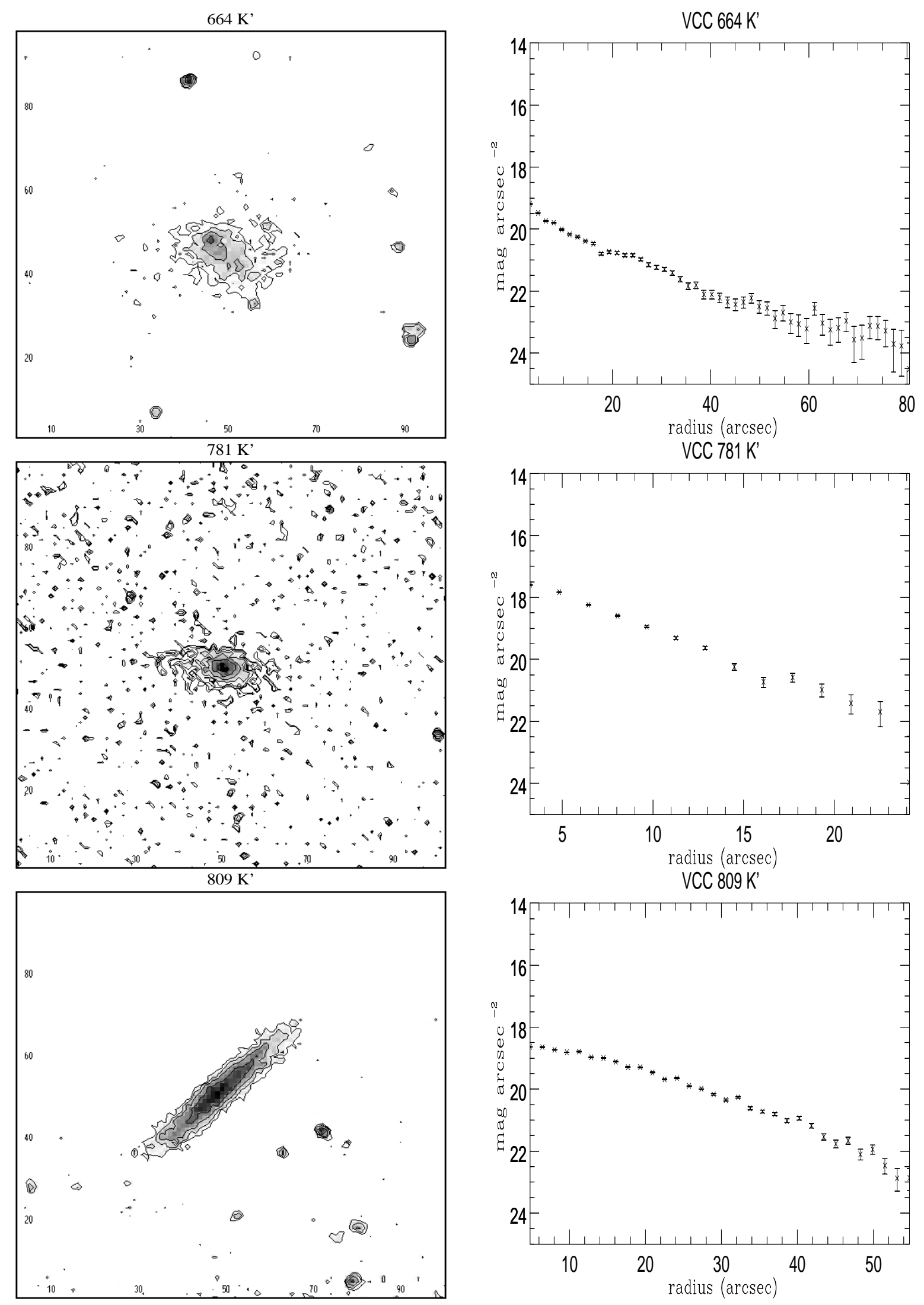

Fig. 3. b) continued 

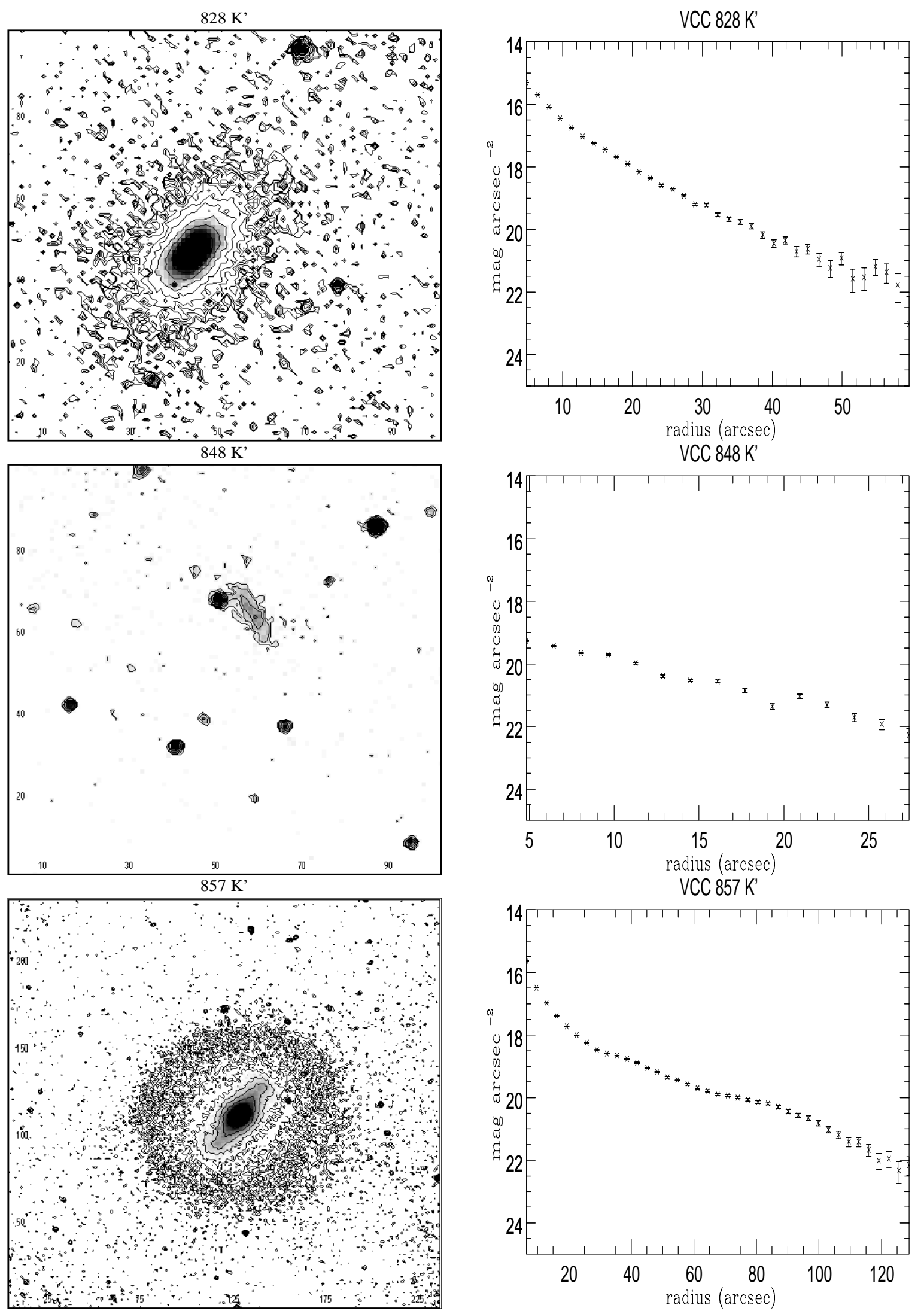

Fig. 3. b) continued 

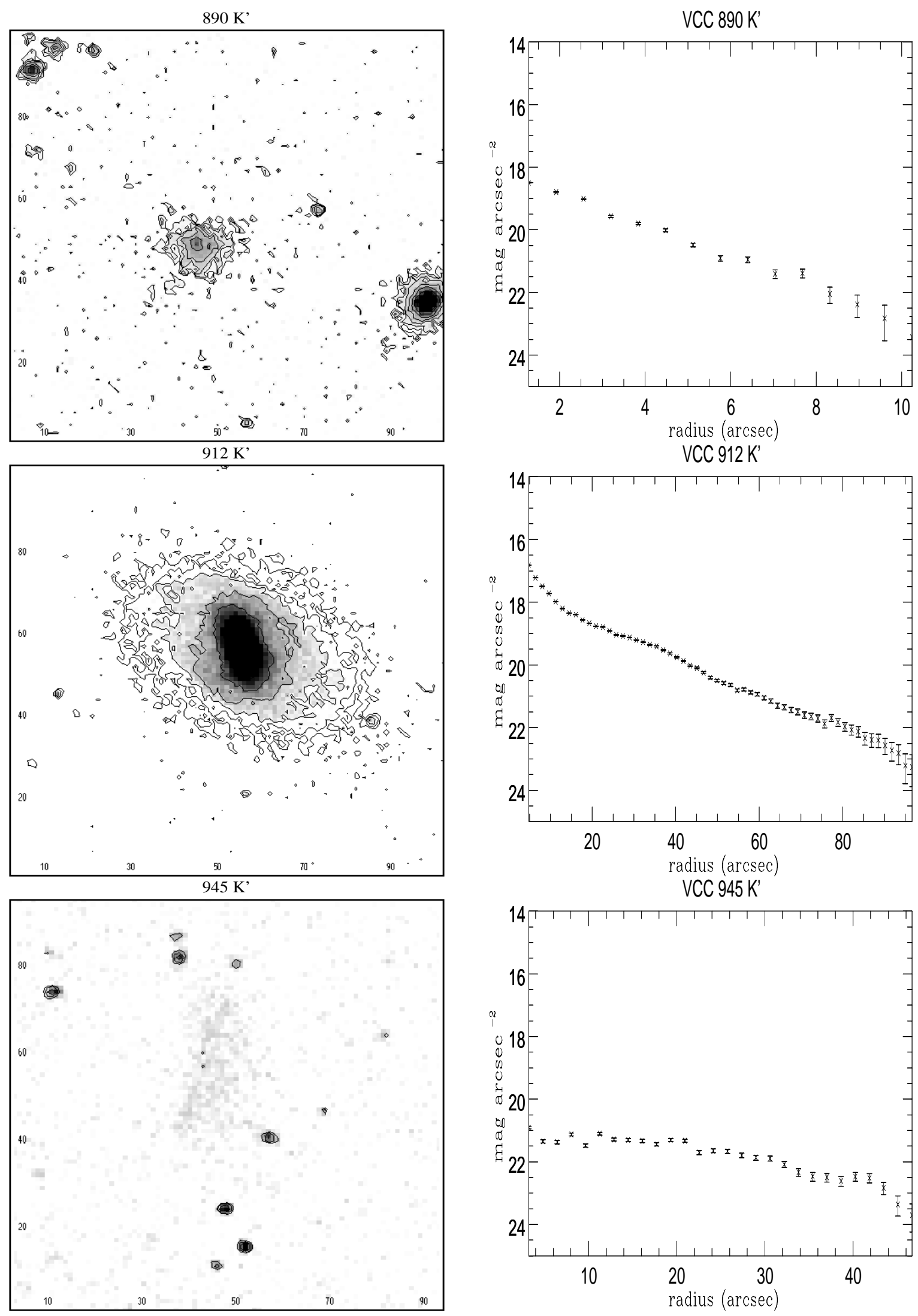

Fig. 3. b) continued 

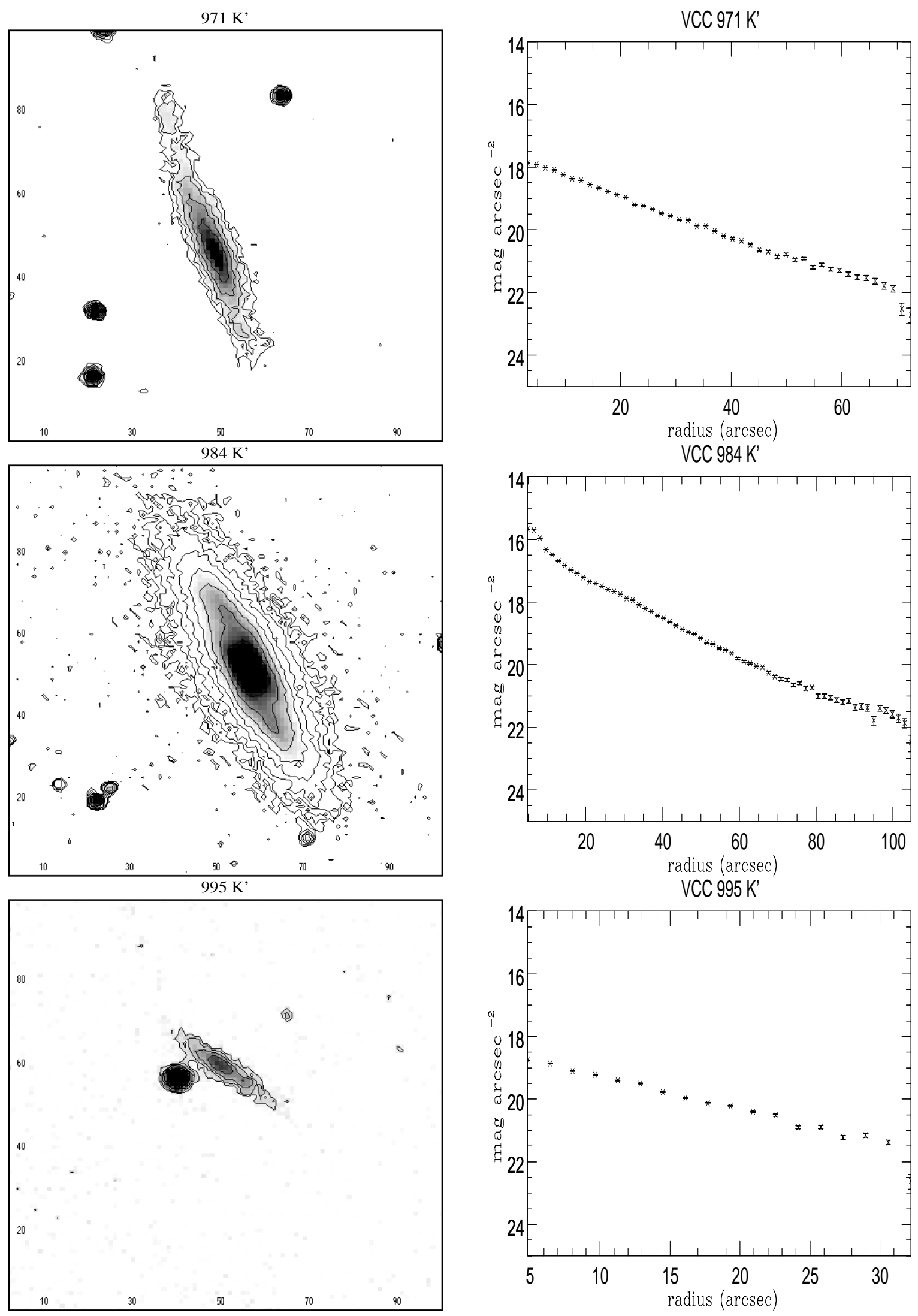

Fig. 3. b) continued 

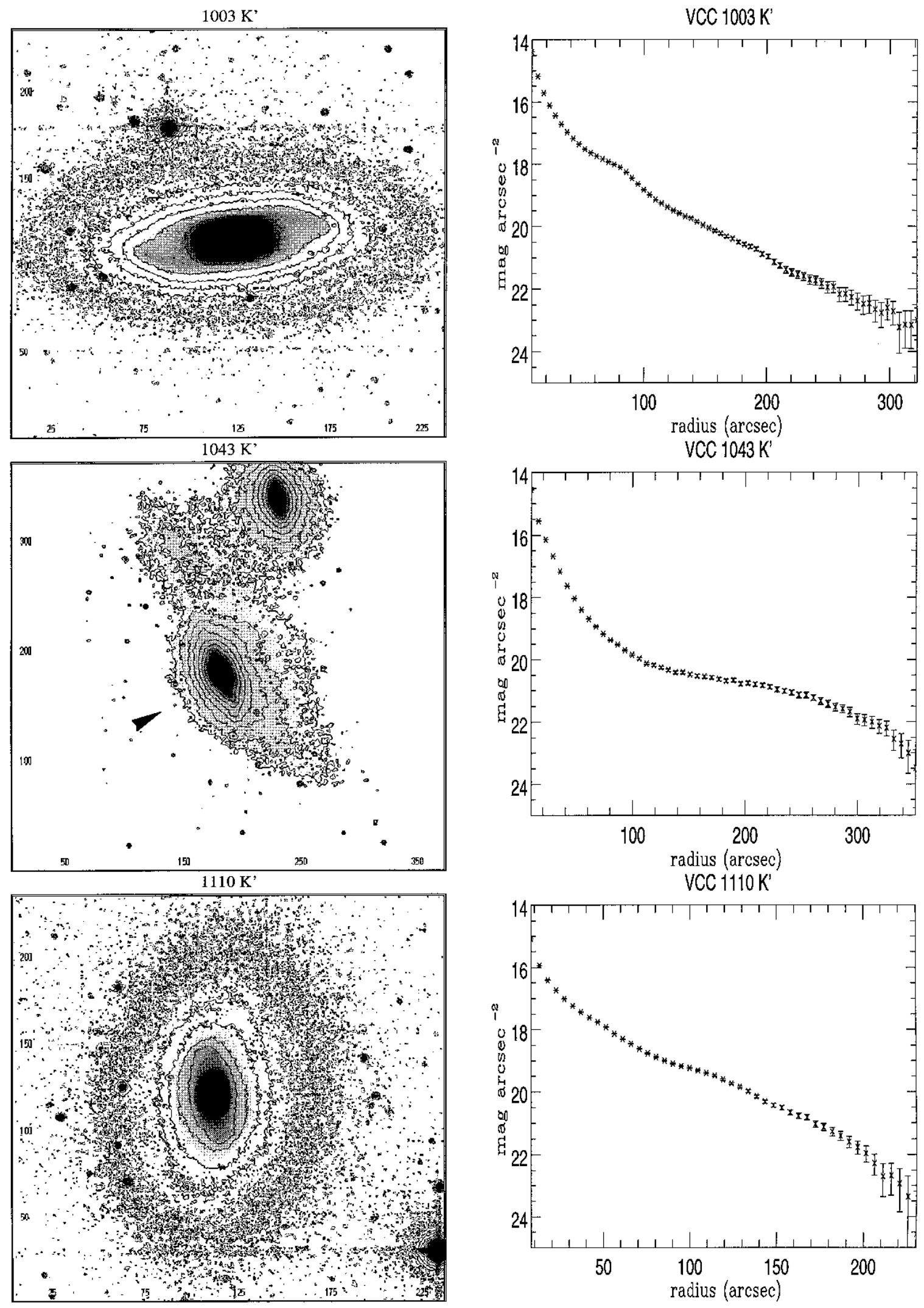

Fig. 3. b) continued 

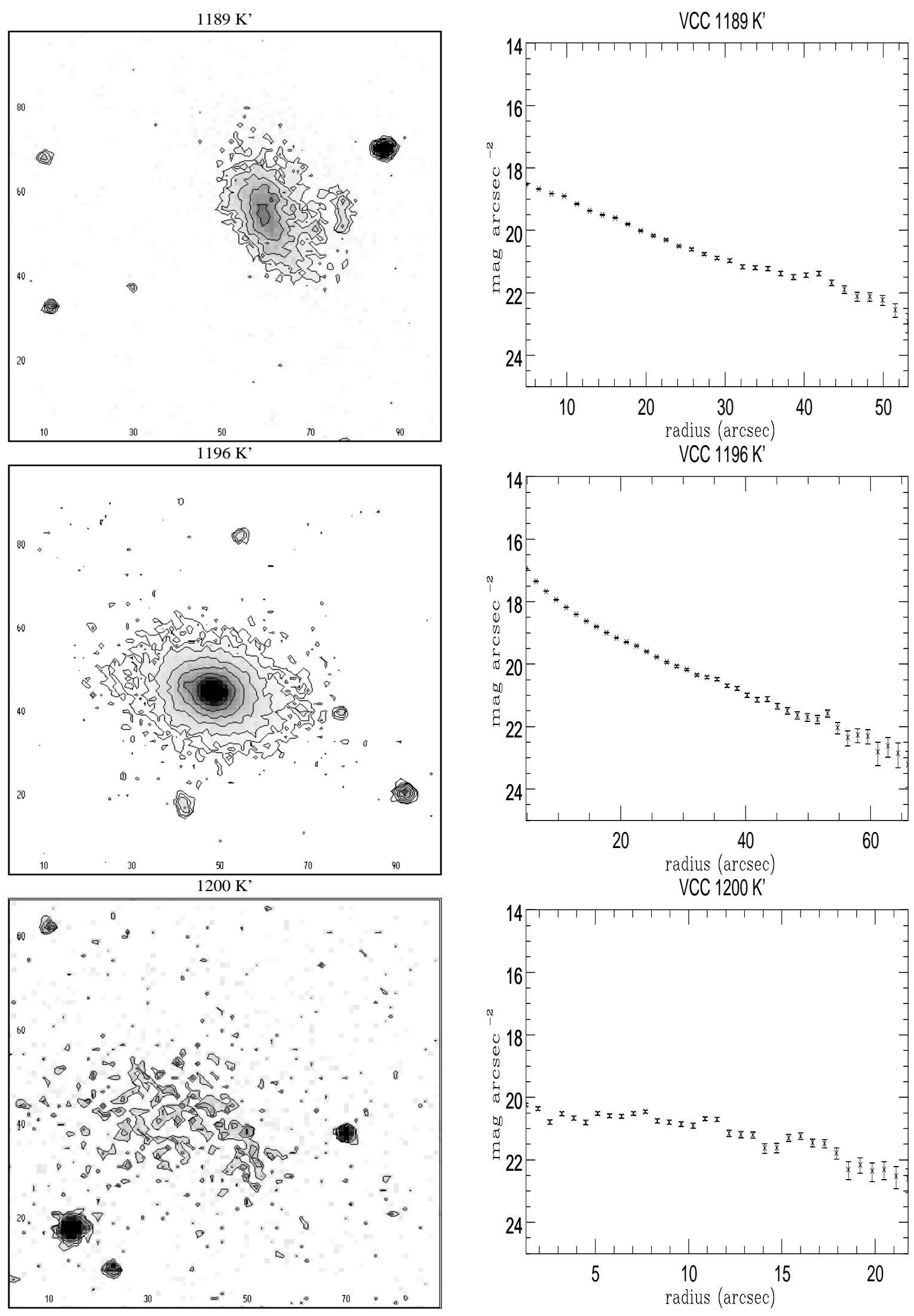

Fig. 3. b) continued 

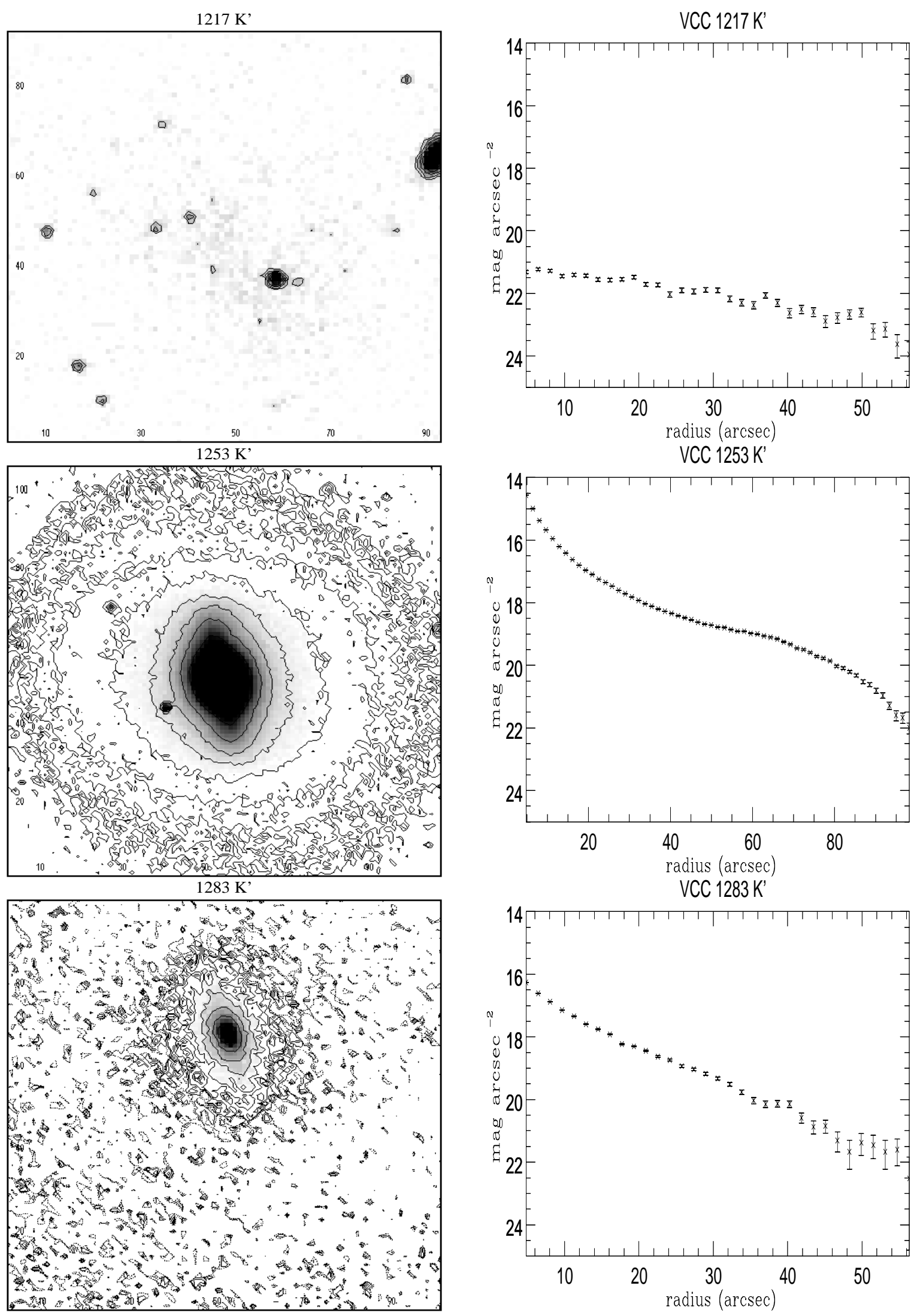

Fig. 3. b) continued 

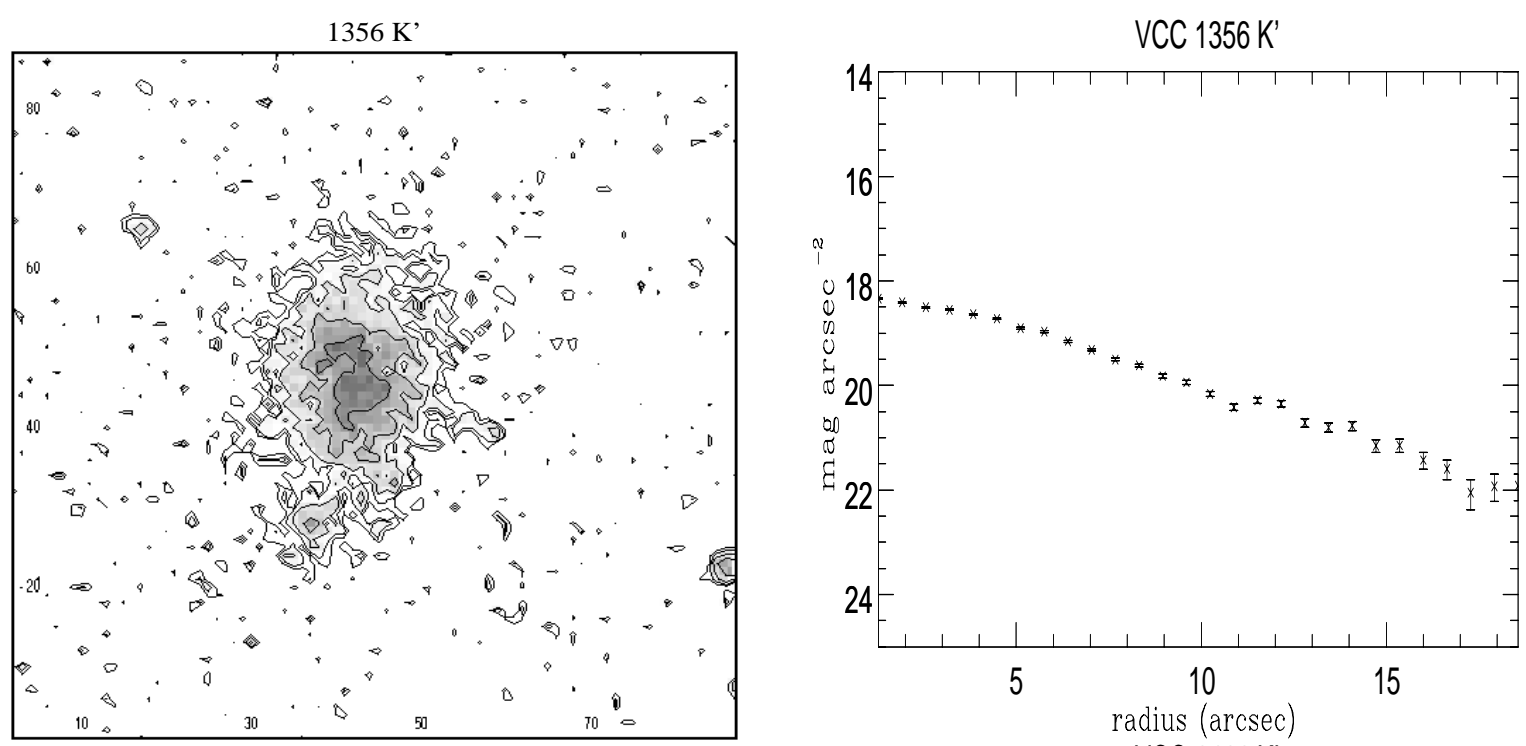

$1411 \mathrm{~K}^{\prime}$
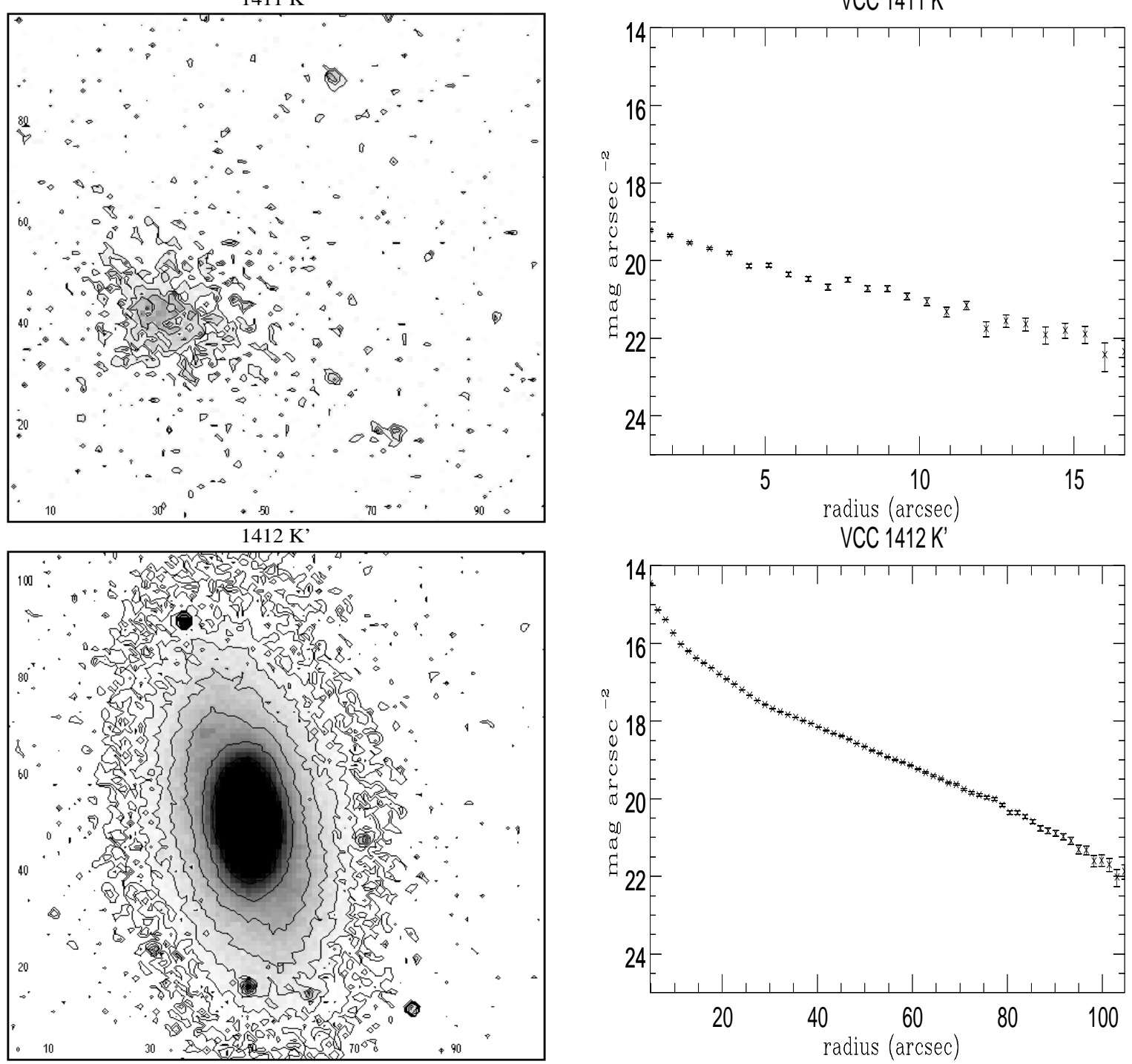

Fig. 3. b) continued 

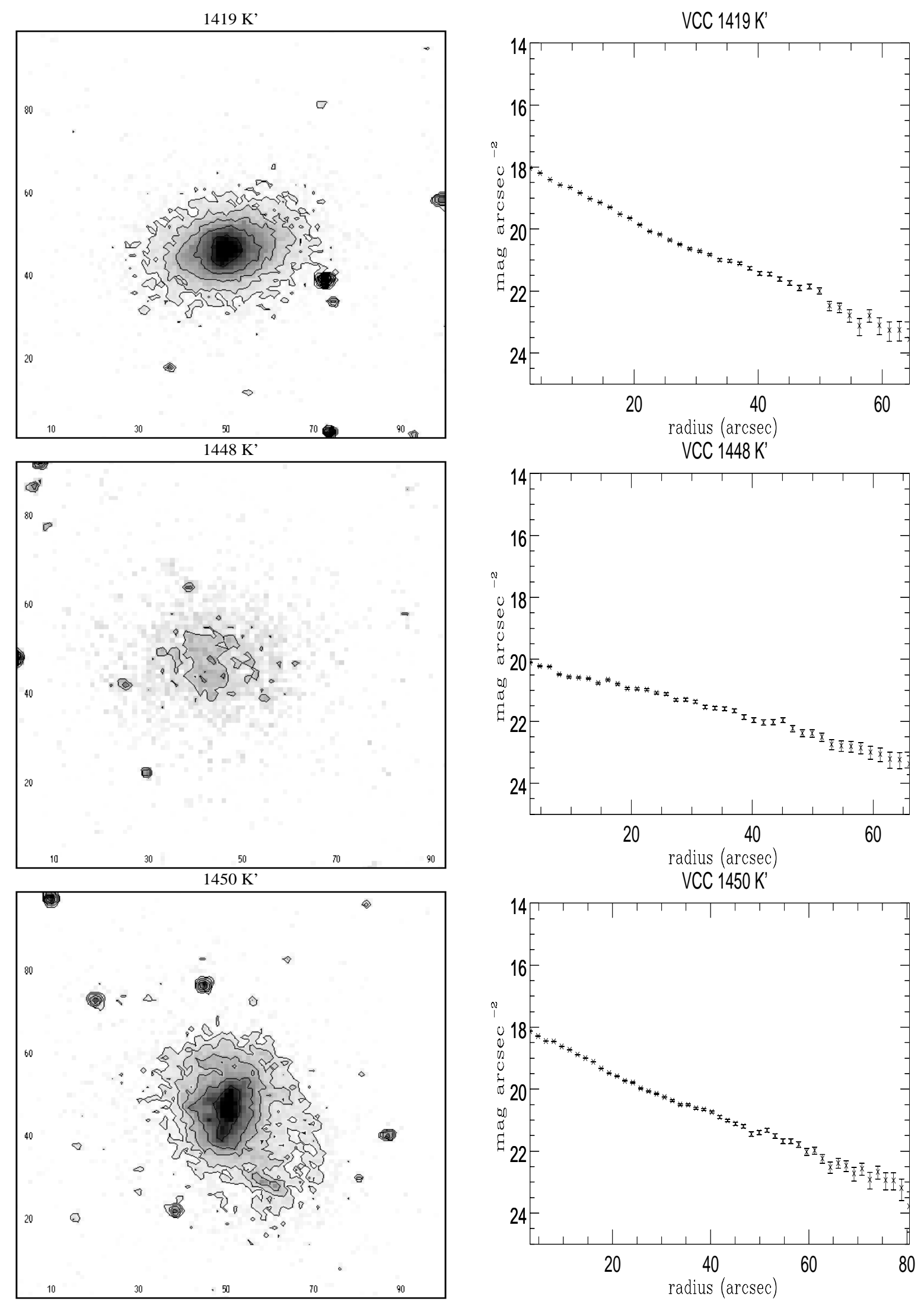

Fig. 3. b) continued 

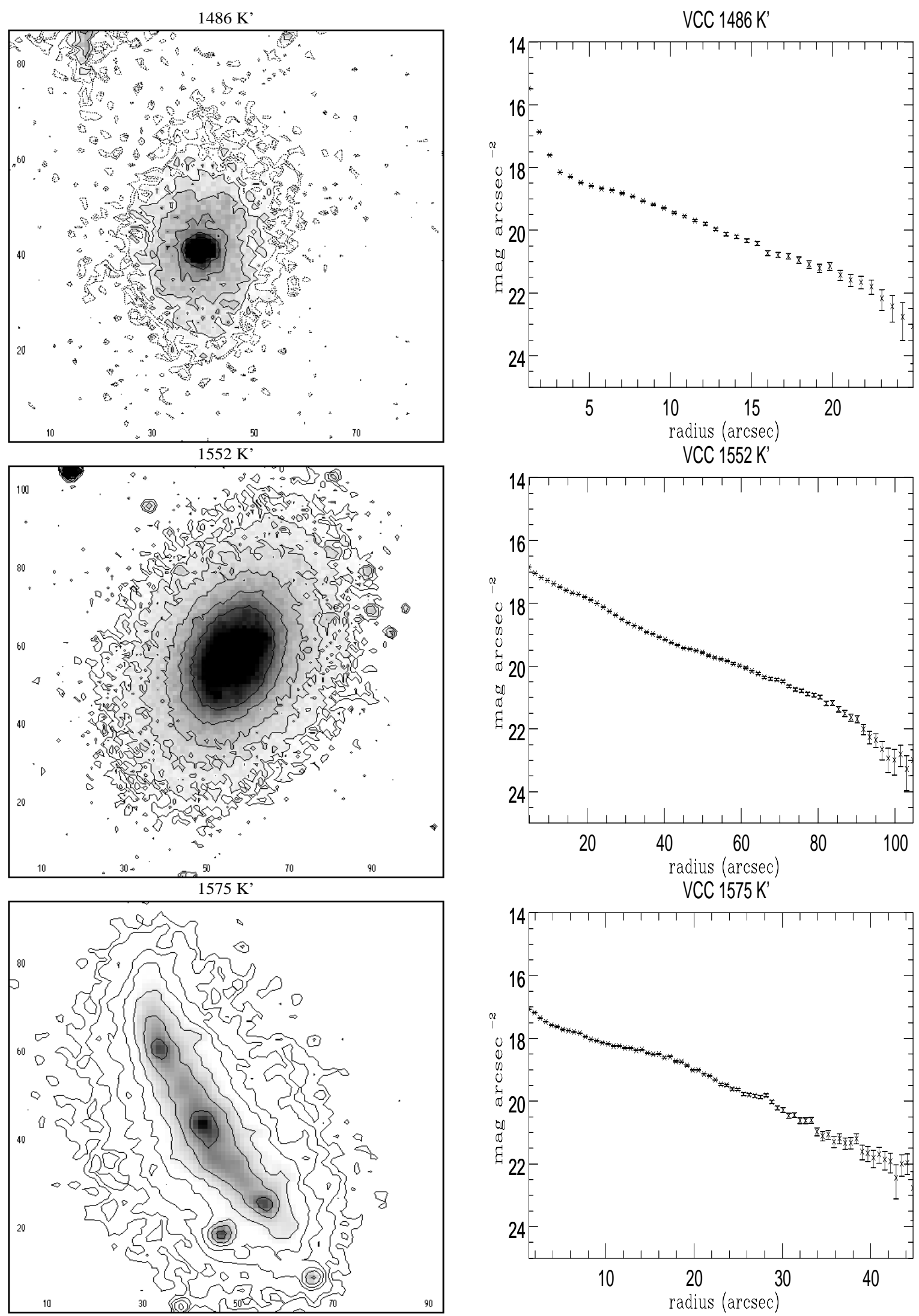

Fig. 3. b) continued 

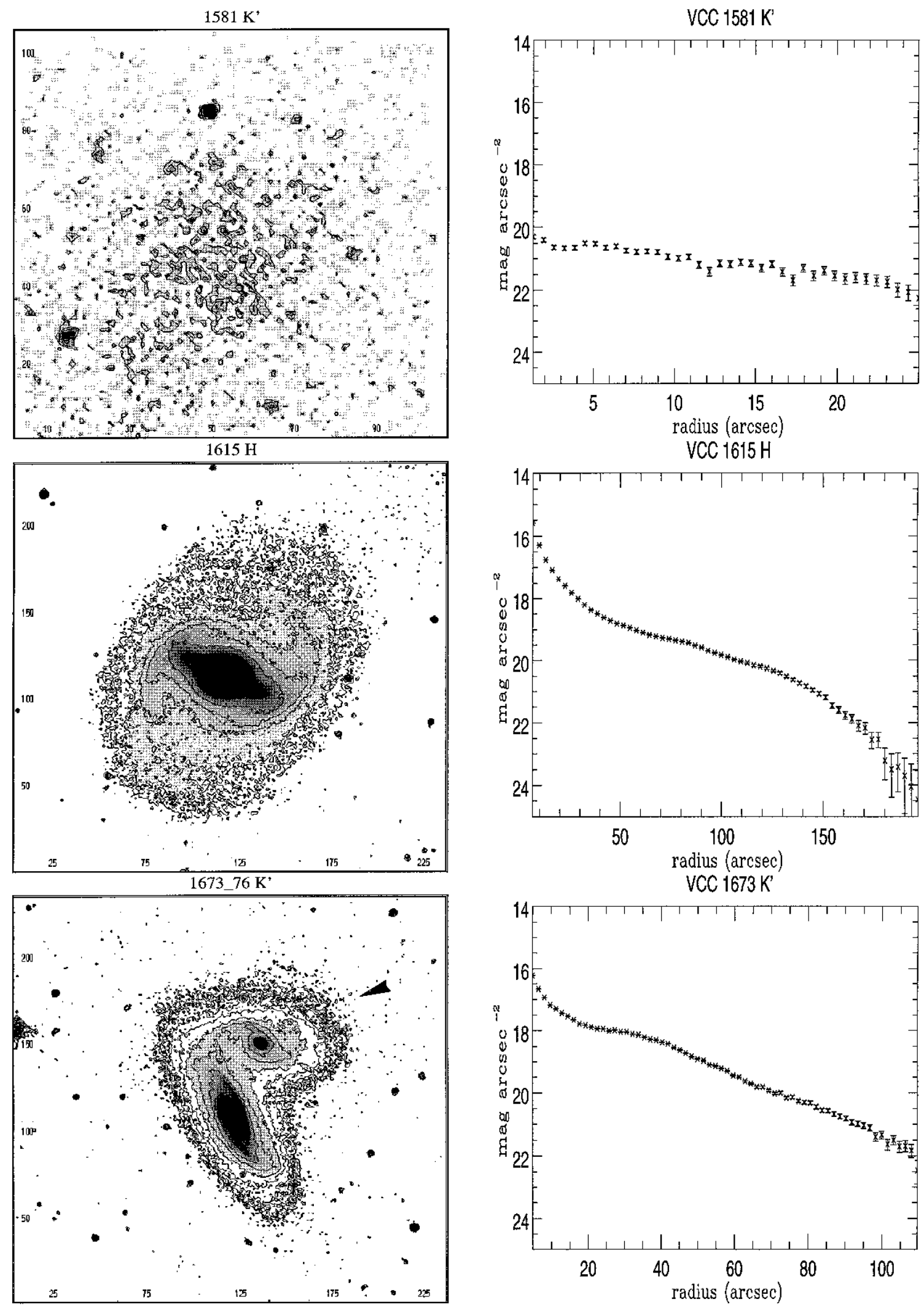

Fig. 3. b) continued 

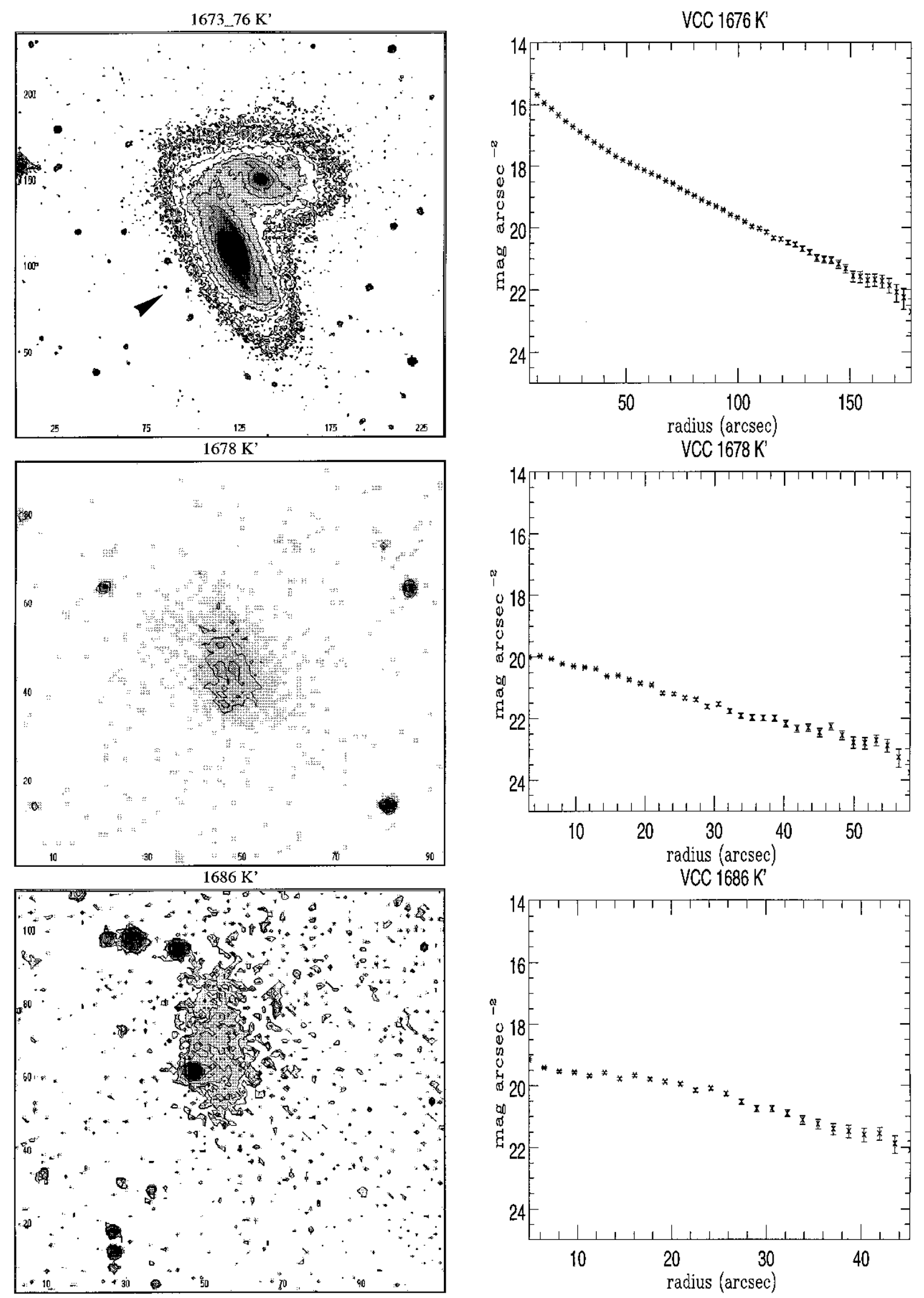

Fig. 3. b) continued 
A. Boselli et al.: NIR surface photometry of Virgo galaxies
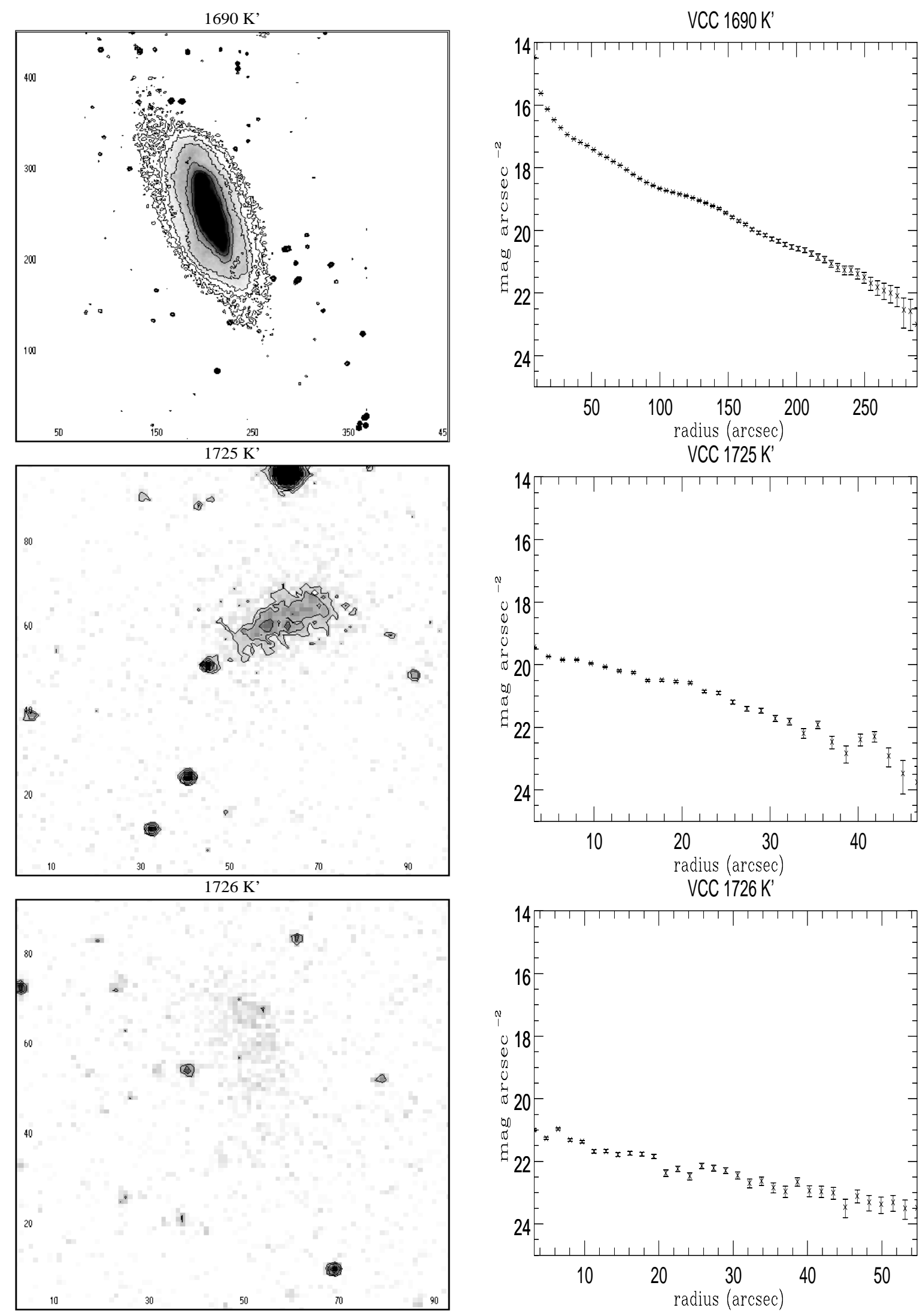

Fig. 3. b) continued 

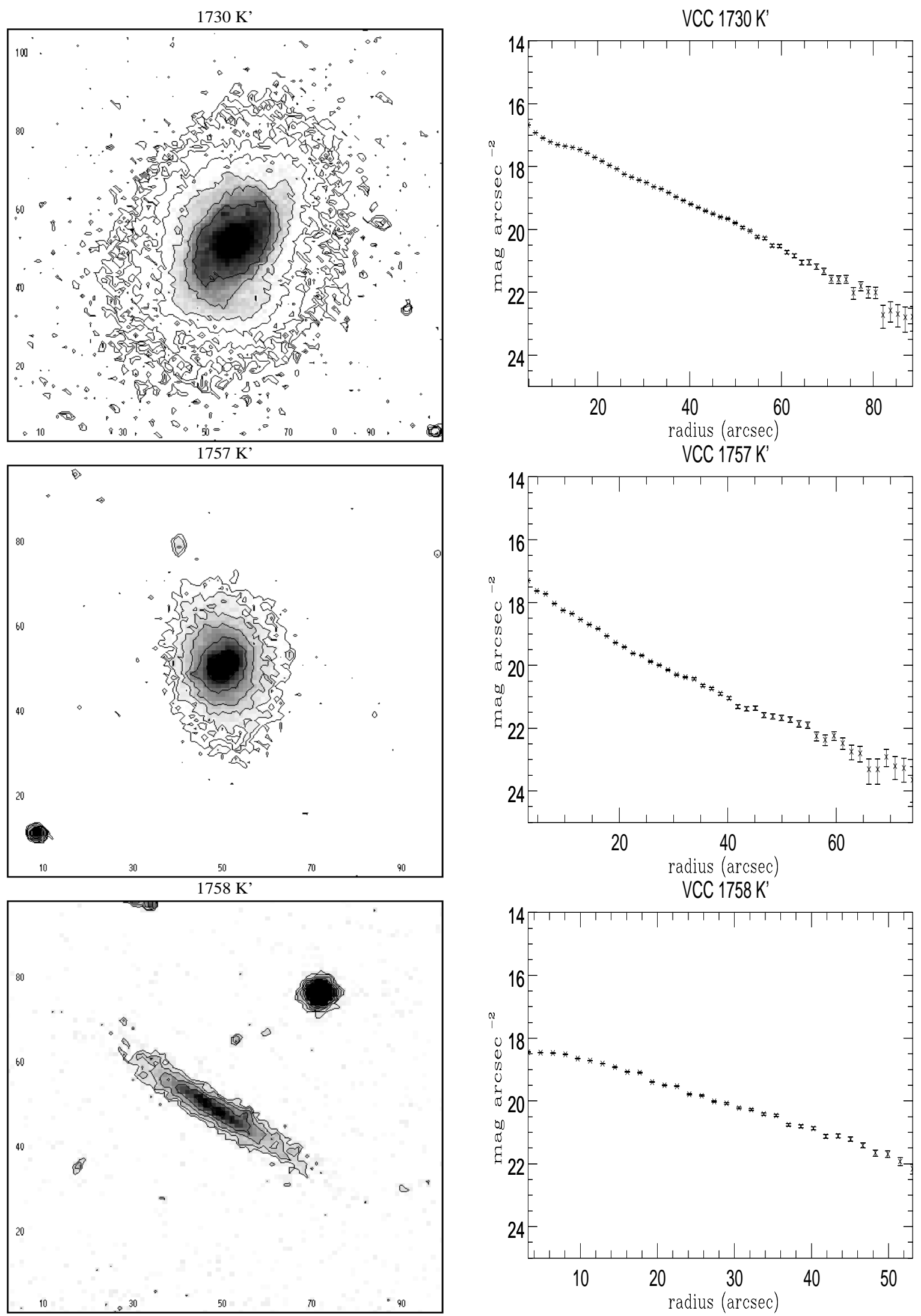

Fig. 3. b) continued 

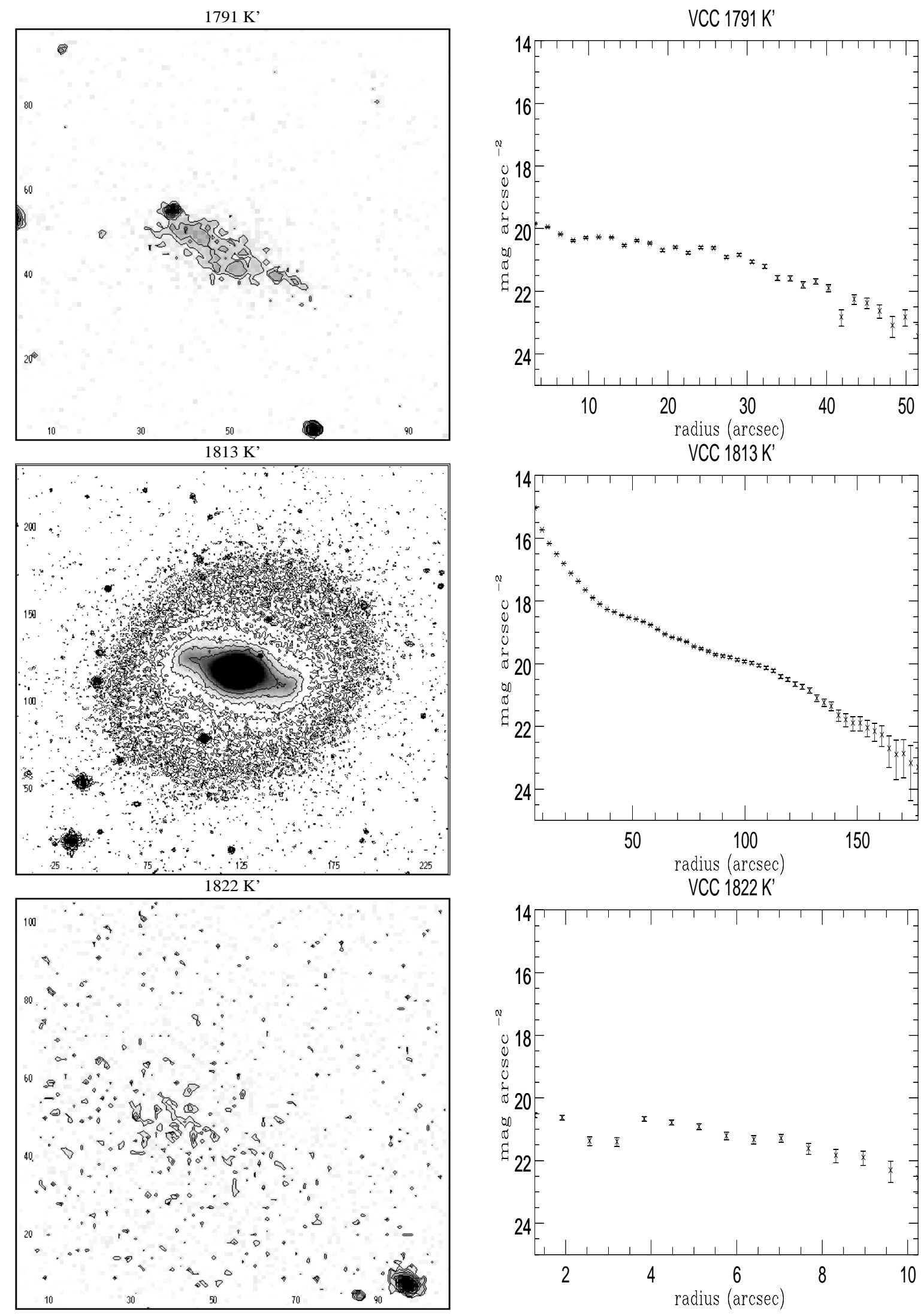

Fig. 3. b) continued 

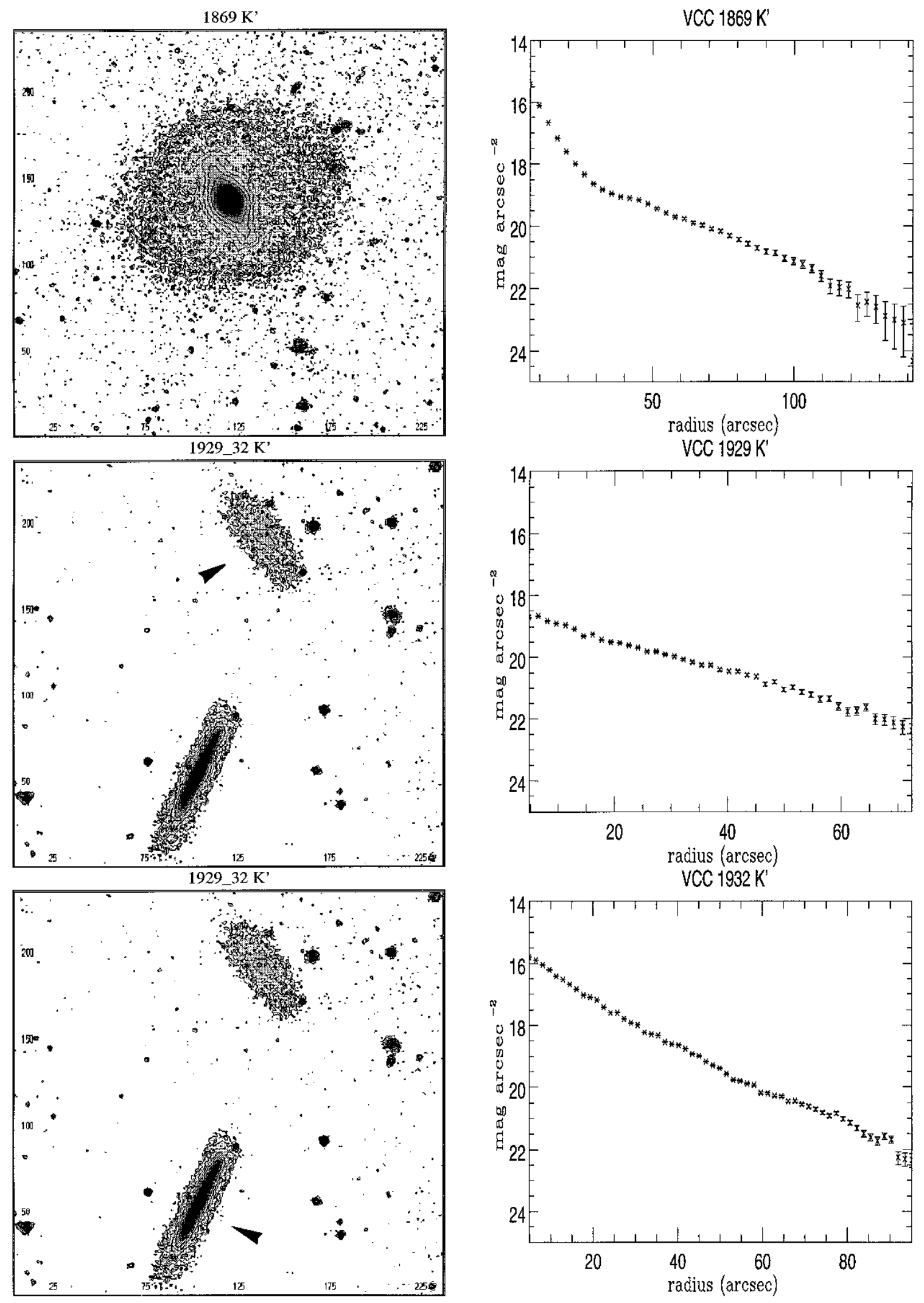

Fig. 3. b) continued 

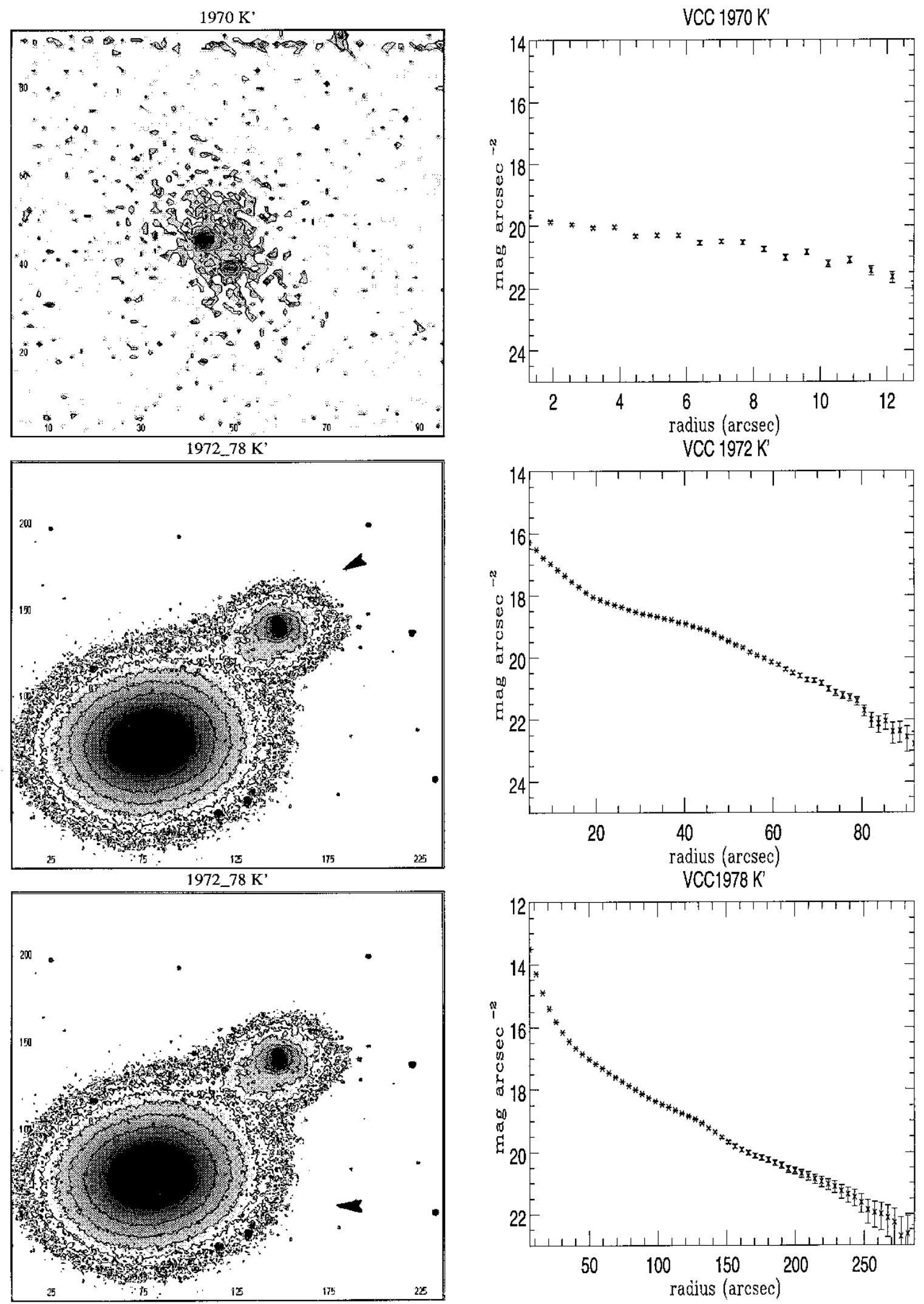

Fig. 3. b) continued 
1999 K'
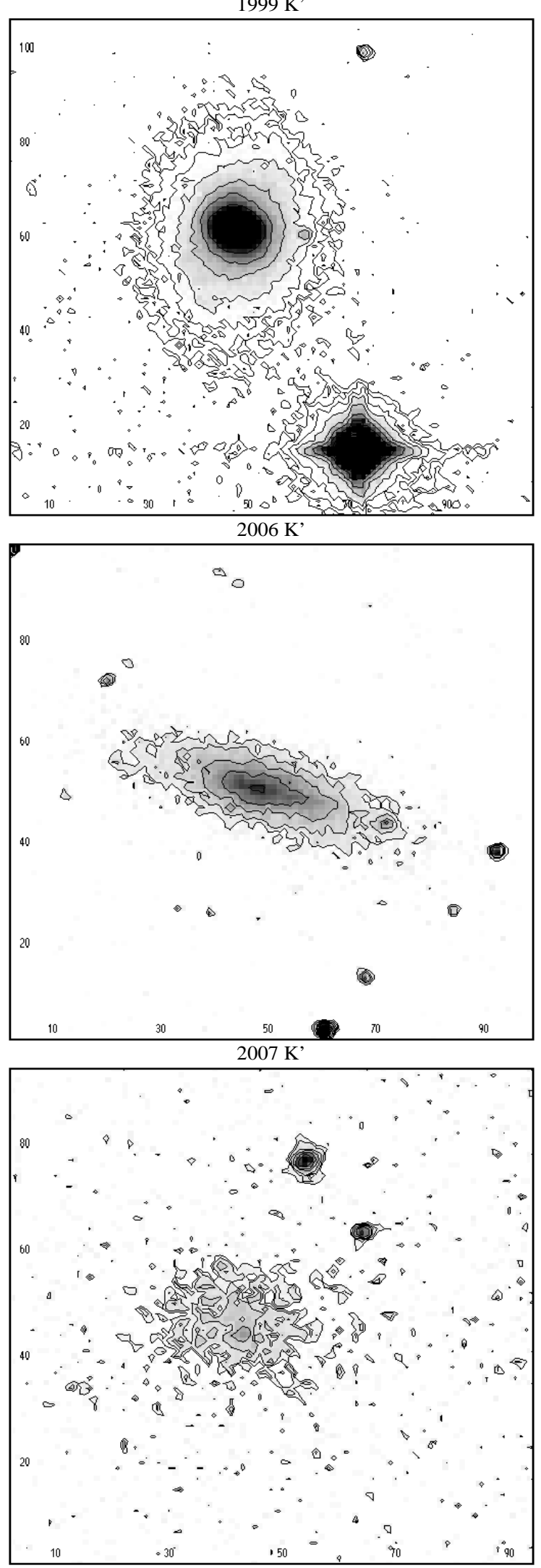

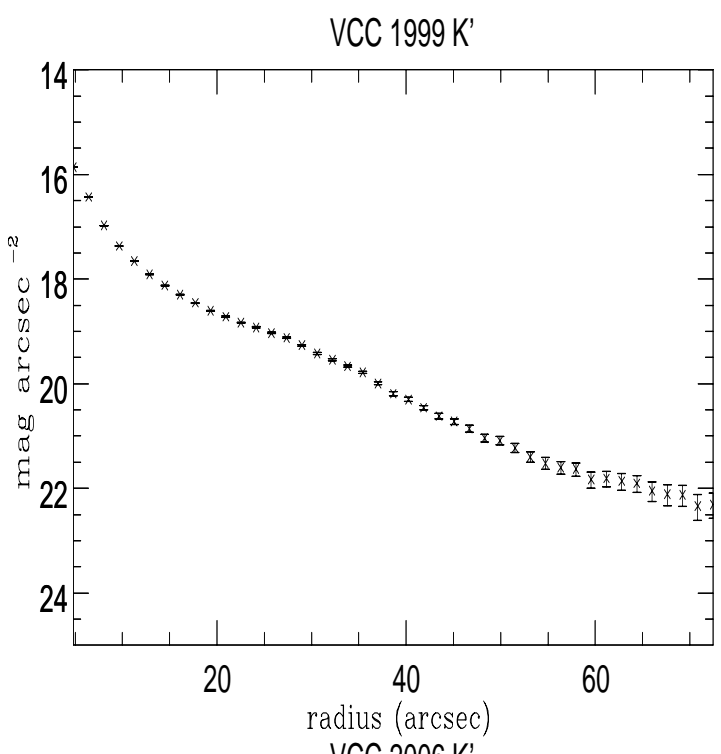

VCC 2006 K'
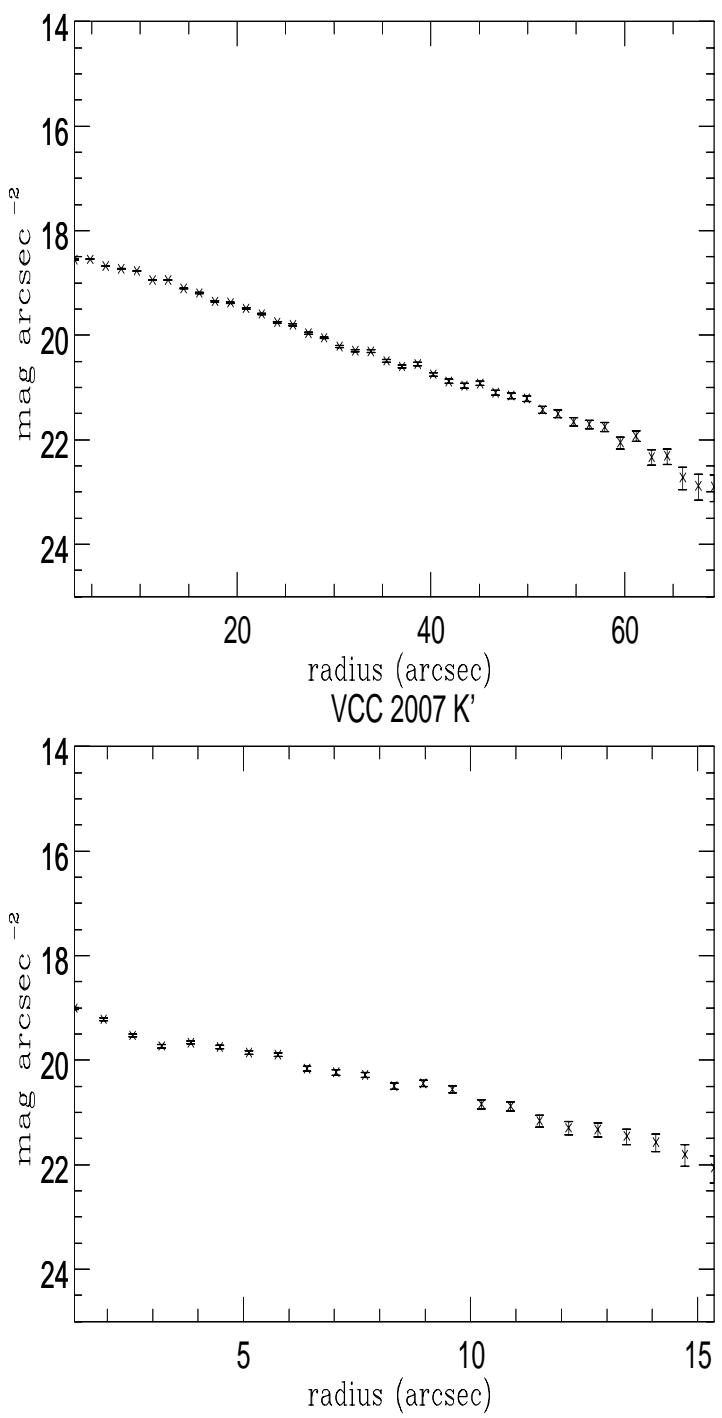

Fig. 3. b) continued 

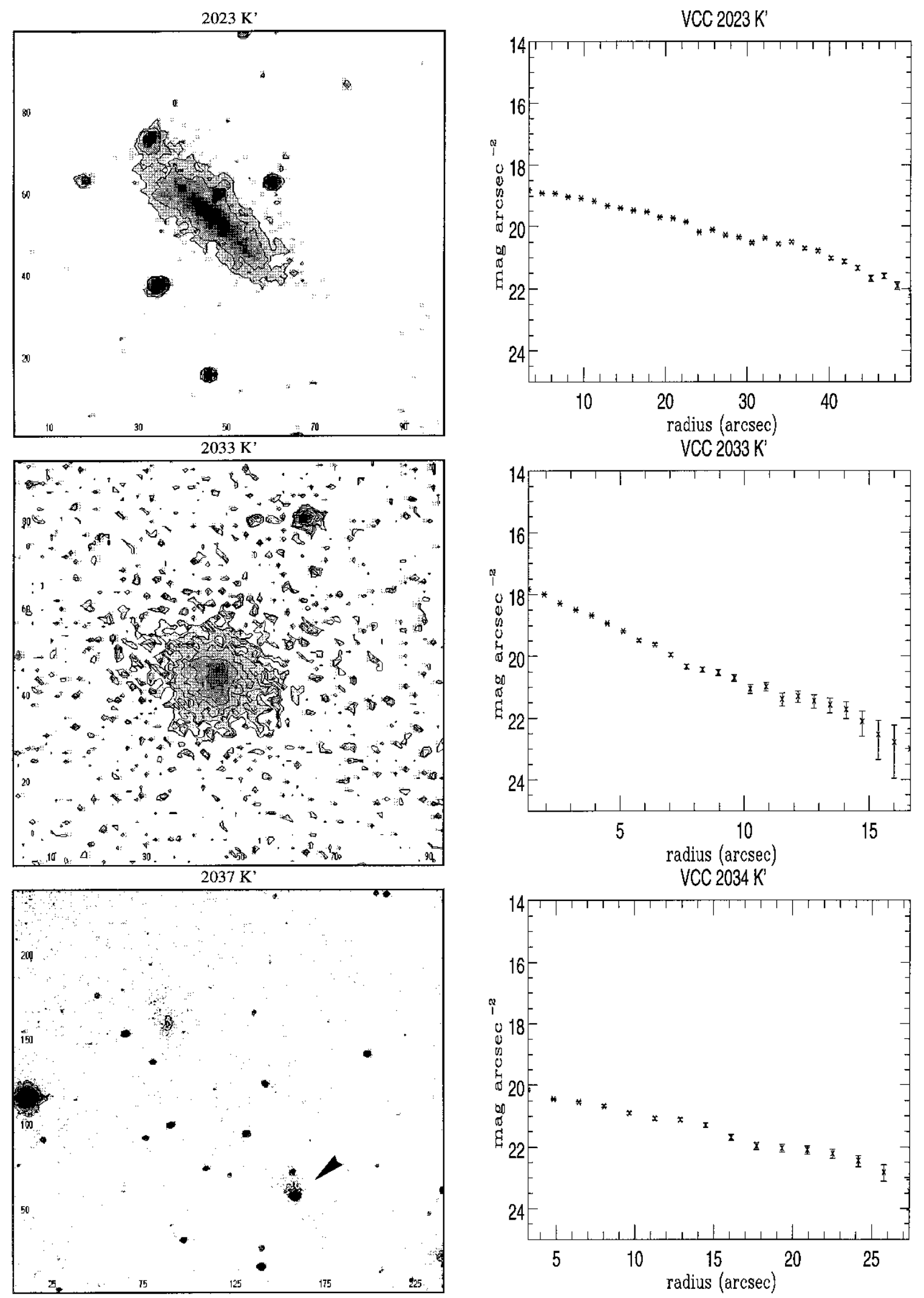

Fig. 3. b) continued 

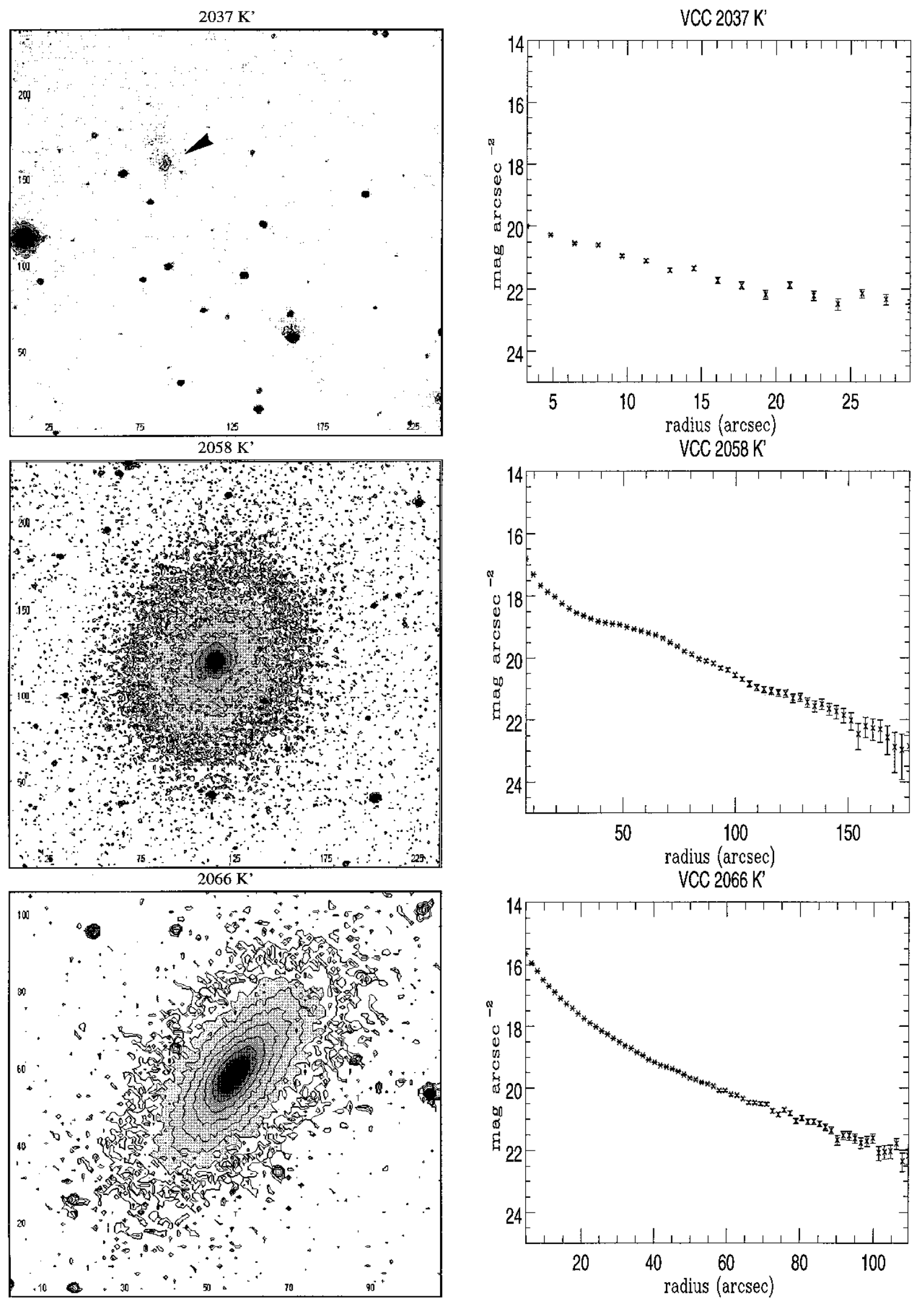

Fig. 3. b) continued 

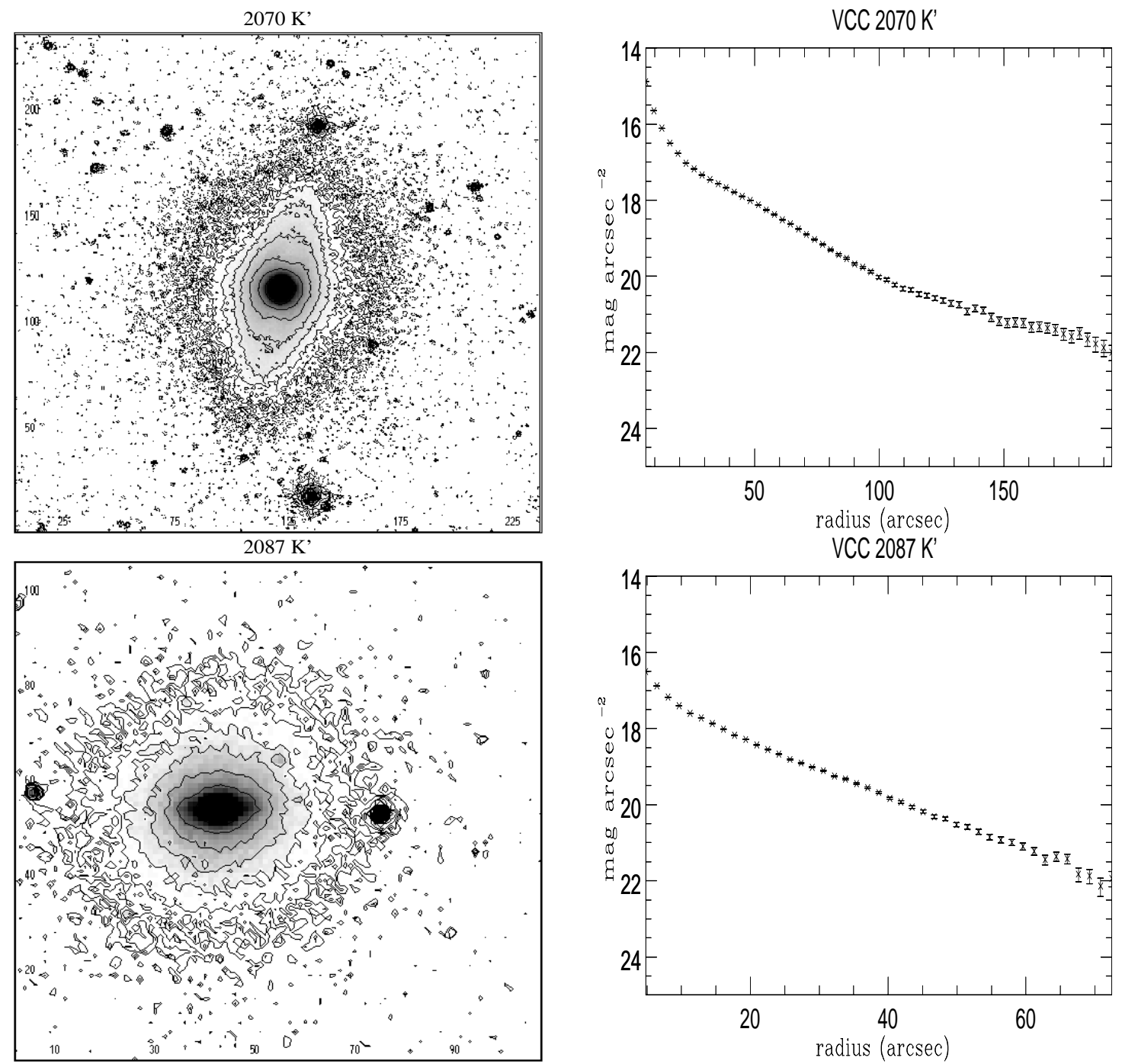

Fig. 3. b) continued 

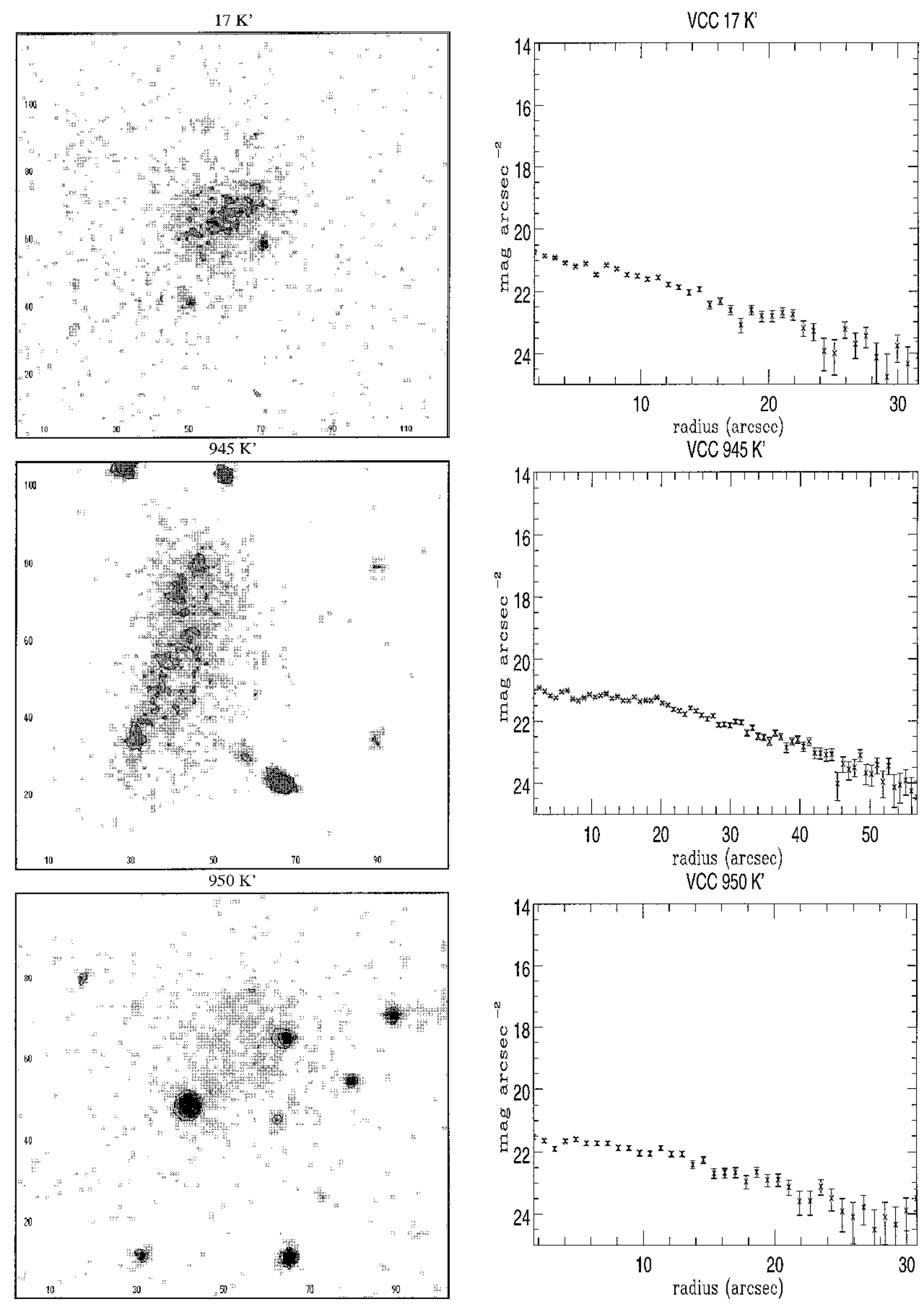

Fig. 3. c) continued 

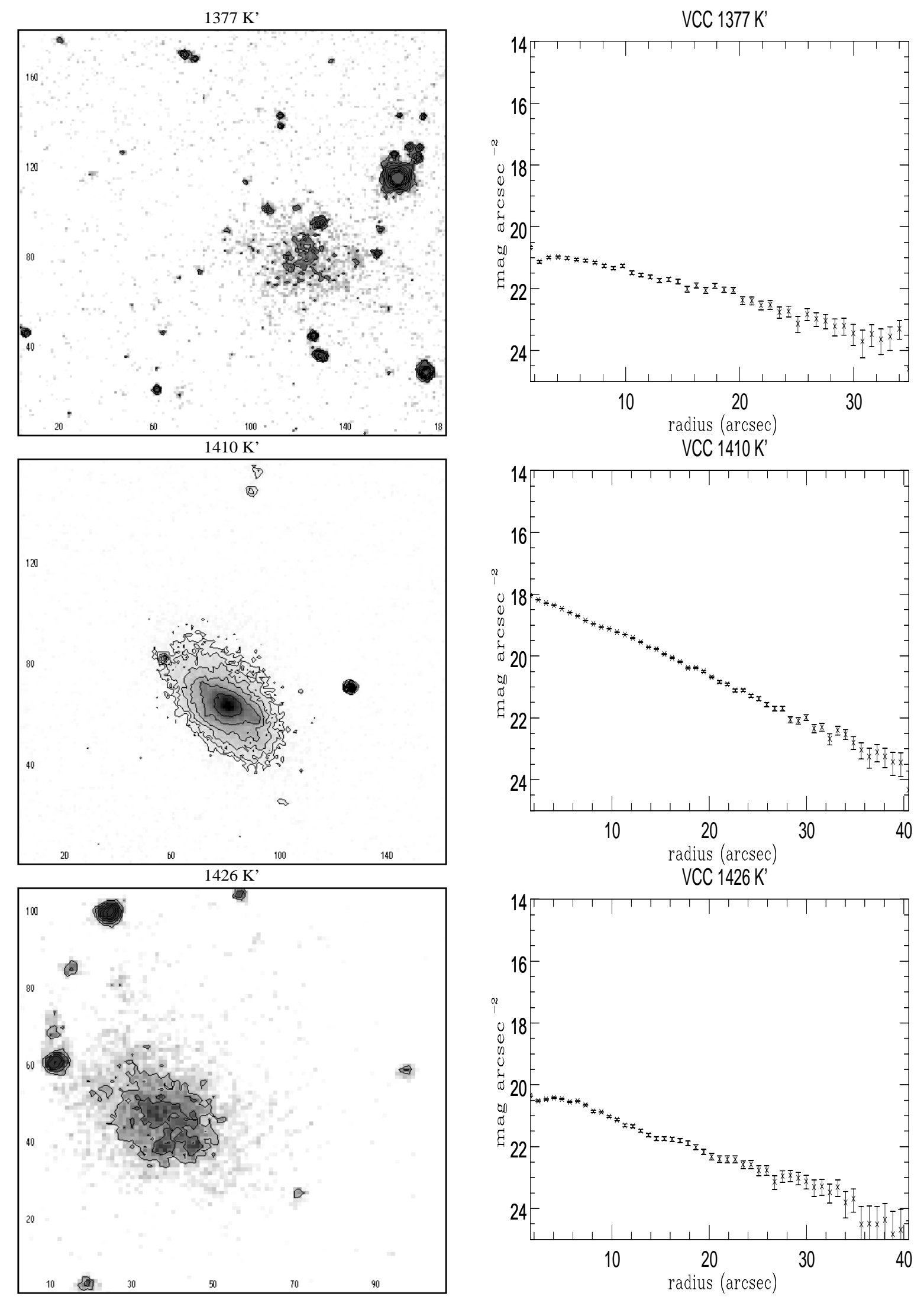

Fig. 3. c) continued 

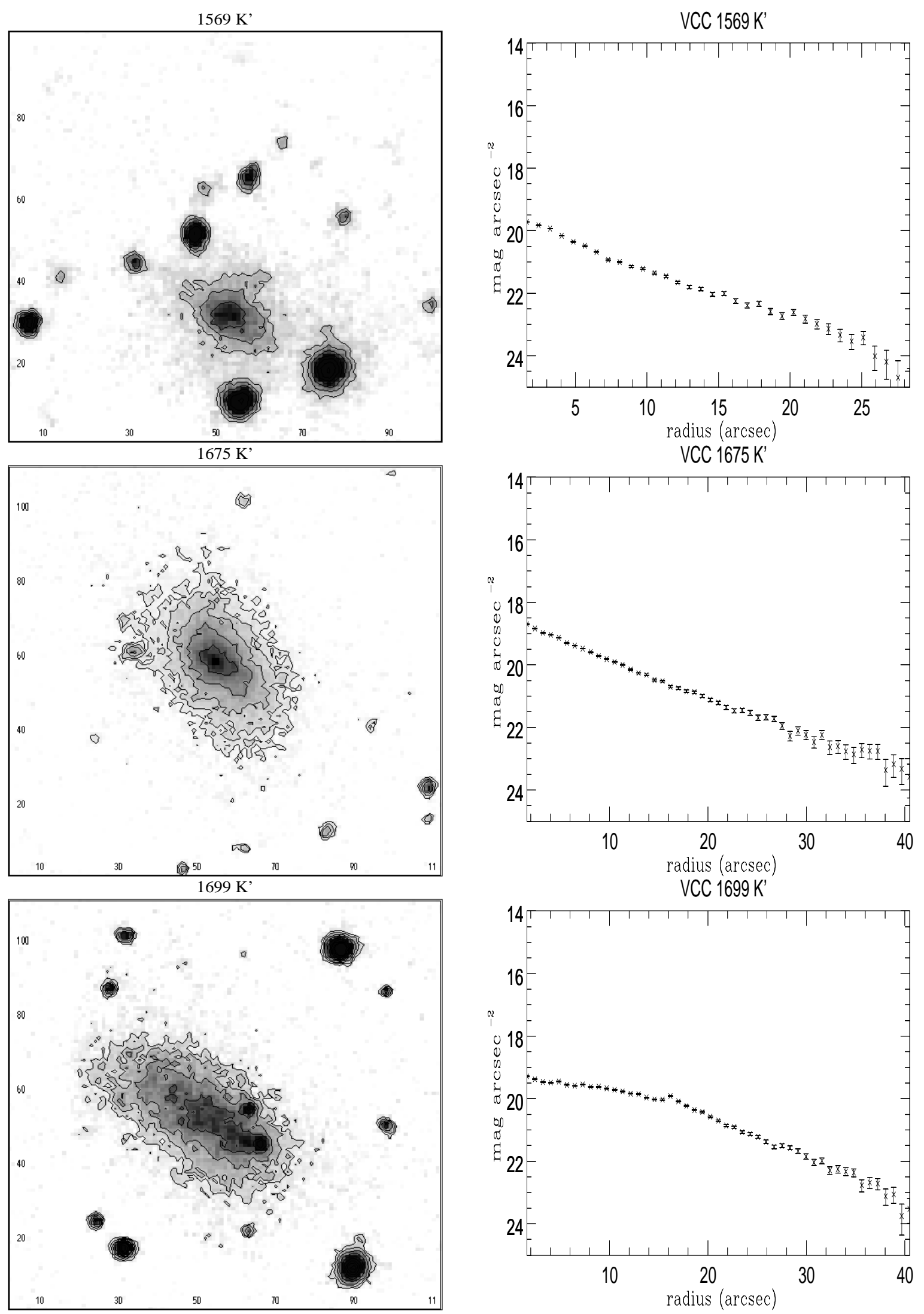

Fig. 3. c) continued 

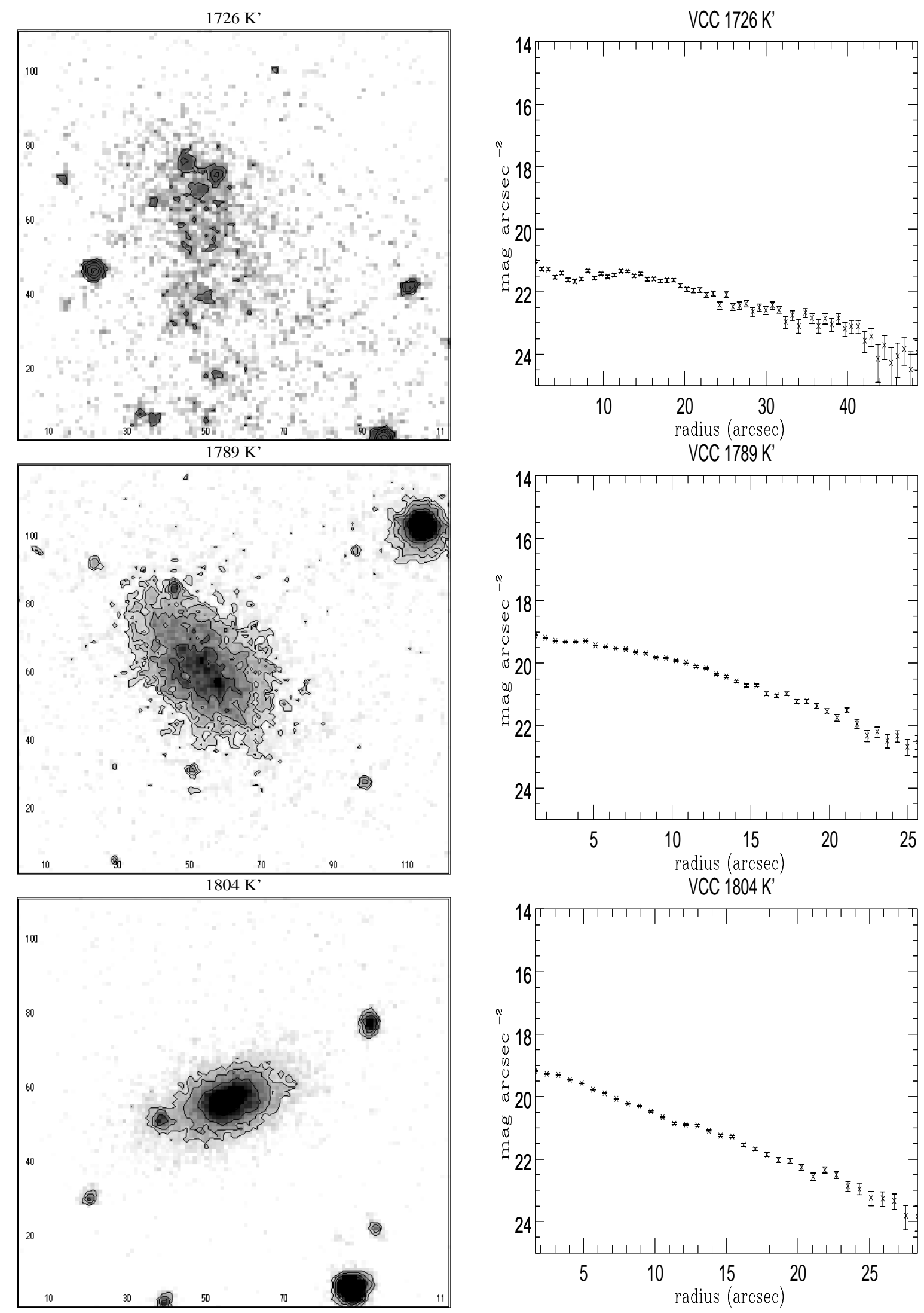

Fig. 3. c) continued 

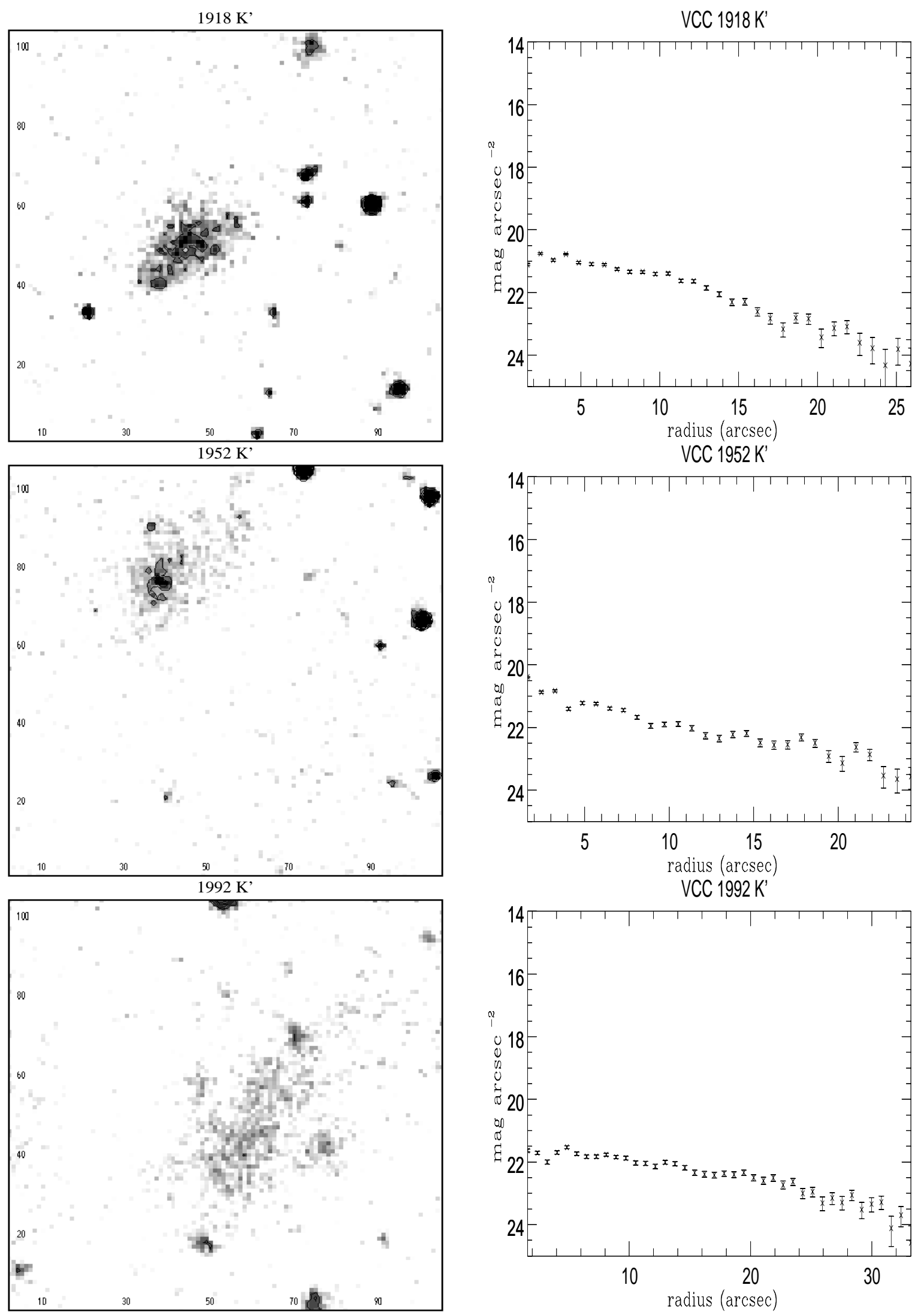

Fig. 3. c) continued 
which imply that the present NIR observations cover a substantial fraction of the optical size of the galaxies.

Figure 5 shows that the ratio between the optical $\left(D_{25.5}(B)\right)$ to NIR $\left(2 \times a_{22.0}\left(K^{\prime}\right)\right)$ diameters correlates with the $B-K^{\prime}$ colour index, (bluer galaxies have larger optical-to-NIR ratios) (given the uncertainty on the $B$ photographic magnitudes, the estimated error on the $B-K^{\prime}$ colour index is $\sim 0.3 \mathrm{mag}$ ). Since the ratio of the optical to NIR surface brightness is not constant in galaxies of different luminosity and colour (Gavazzi et al. 1996c), the trend observed in Fig. 4 is not entirely due to the non-linearity between the optical and NIR diameters.

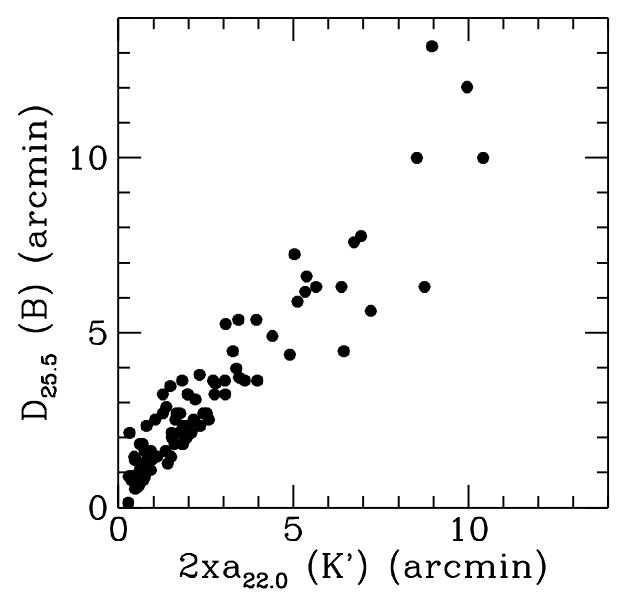

Fig. 4. Relation between $K^{\prime}$ band diameters at the $22 \mathrm{mag} \operatorname{arcsec}^{-2}$ isophote determined in this work and the optical $B$ diameters from Binggeli et al. (1985)

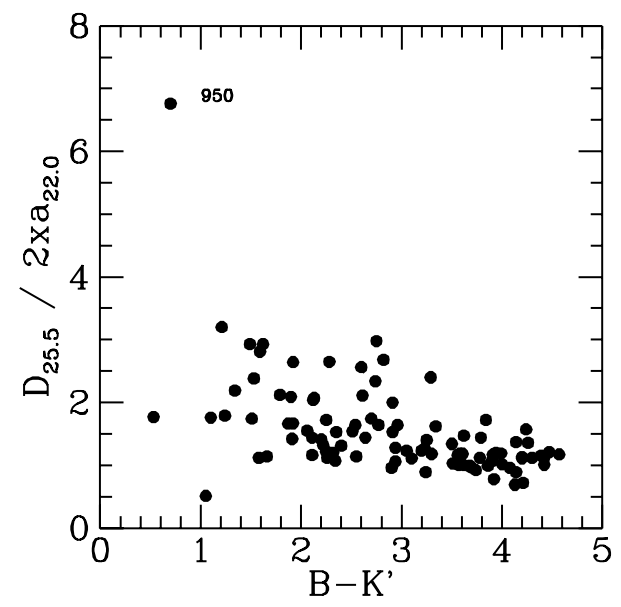

Fig. 5. The relation between the optical $\left(D_{25.5}(B)\right)$ to NIR $\left(2 \times a_{22.0}\left(K^{\prime}\right)\right)$ diameter ratio versus the $B-K^{\prime}$ colour index

This result indicates that in the red galaxies, which are generally bright spirals (see Sect. 4.2), the old and young stellar components are distributed on discs of comparable dimensions. In the bluest objects, which are generally dwarfs, young stars are characterized by an higher surface density, thus by a larger isophotal diameter, than the old stellar component.

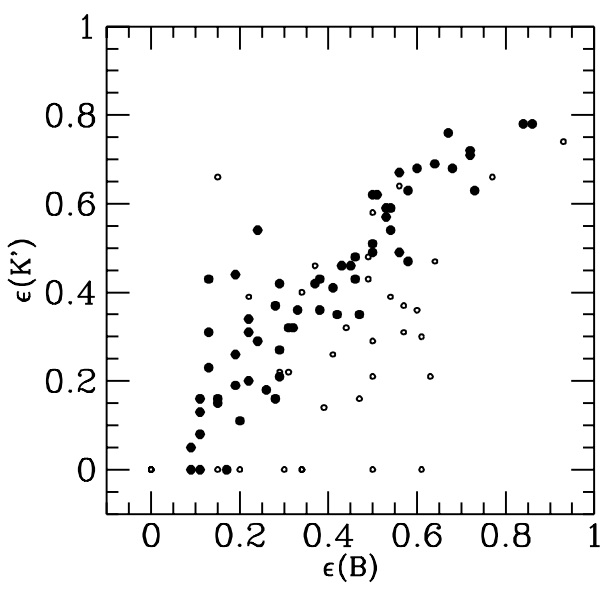

Fig. 6. Relation between the ellipticity $(1-b / a)$ in the $K^{\prime}$ band determined in this work and the corresponding ellipticity derived in the visible. Galaxies with $K^{\prime}<12.0$ mag are marked with filled dots, open dots indicate objects with $K^{\prime}>12.0 \mathrm{mag}$

The relation between the ellipticity determined in the $K^{\prime}$ band and the one determined in the $B$ band by Binggeli et al. (1985) is given in Fig. 6. Two different symbols are used for bright $\left(K^{\prime}<12.0 \mathrm{mag}\right)$ and weak $\left(K^{\prime}>12.0 \mathrm{mag}\right)$ objects. As expected, there is a better agreement for bright galaxies, while a large scatter is observed for dwarf irregulars and blue compact objects, for which ellipses are just a crude representation of their shape. Part of the scatter is probably due to the different techniques used to determine the ellipticity by Binggeli et al. (1985) and by us: while in the $B$ band the ellipticity is calculated from the ratio of the minor to the major diameter at $25.5 \mathrm{mag} \operatorname{arcsec}^{-2}$ isophote, in the NIR it is given by the ellipse which best fits the $K^{\prime} 21.0 \mathrm{mag} \operatorname{arcsec}^{-2}$ isophote of the galaxy.

The relation between the uncorrected colour index $B-K^{\prime}$ and the morphological type is given in Fig. 7, while Fig. 8 shows the relation between the concentration index (at the $22.0 \mathrm{mag} \operatorname{arcsec}^{-2}$ ) and the morphological type.

Galaxies of earliest morphological classes have in general redder $B-K^{\prime}$ colours and higher concentration indices than dwarfs and late type systems, indicating that they are generally dominated by old stars mainly distributed in the bulge and/or in steep discs. The scatter in both relations is however large. Pure exponential discs $\left(C_{31} \leq 3\right.$; Gavazzi et al. 1996c) are found generally in late-type systems, with some exceptions in early spirals. 


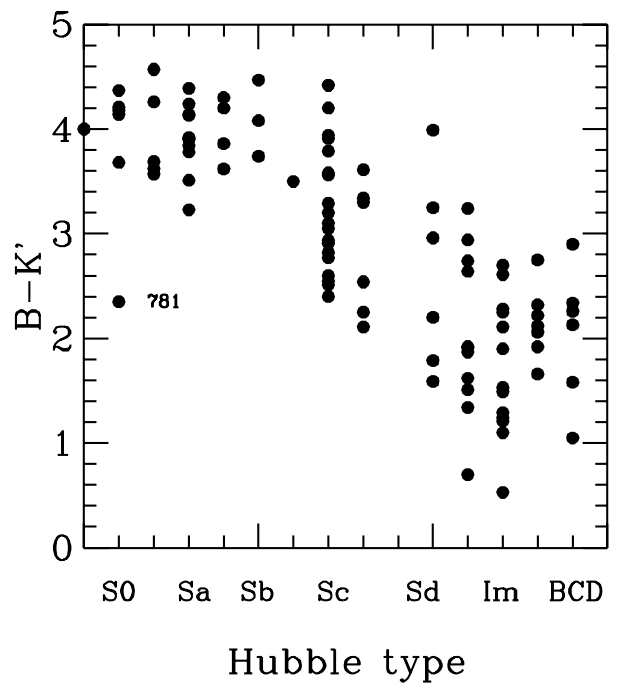

Fig. 7. Relation between the uncorrected colour $B-K^{\prime}$ and the morphological type

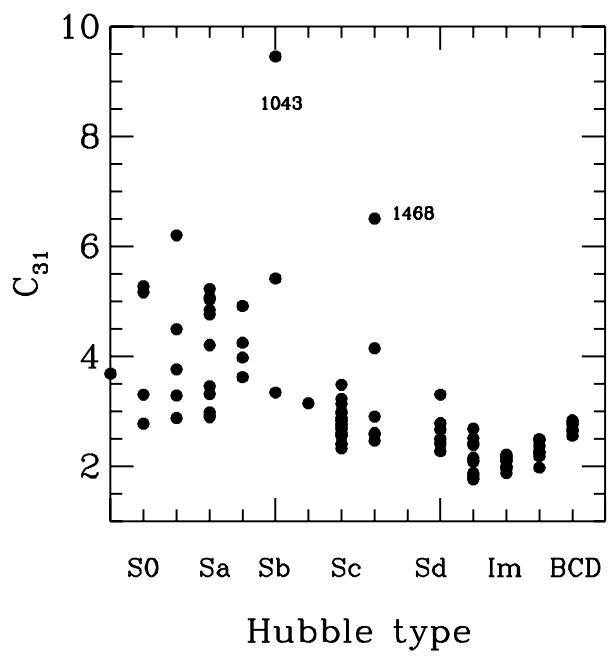

Fig. 8. Relation between the concentration index (at the $22.0 \mathrm{mag} \operatorname{arcsec}^{-2}$ isophote) and the morphological type

This result is in agreement with that found by Gavazzi et al. (1996a,b). It is interesting to note that, although BCD galaxies have $B-K^{\prime}$ colours similar to Sm and Im galaxies, they have higher $K^{\prime}$ concentration indices. This indicates that in BCD galaxies the stars emitting in the NIR bands have a centrally concentrated distribution, as for the blue component.

The relation between the $B-K^{\prime}$ colour and the $K^{\prime}$ magnitude is given in Fig. 9. Since the galaxies are at the same distance, this relation corresponds to a colour - absolute magnitude relation. Furthermore, as shown by Gavazzi (1993) and Gavazzi et al. (1996c), the NIR absolute magnitude is a good indicator of the total mass of galaxies. Figure 9 thus indicates that brighter and more massive galaxies have redder colours than low mass ob-

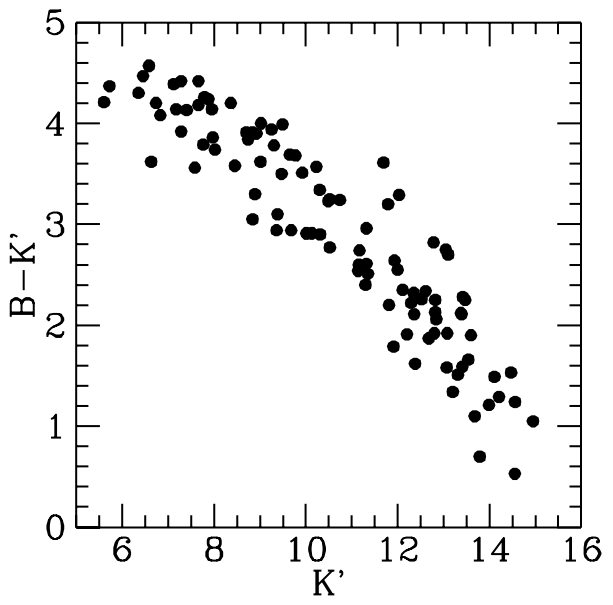

Fig. 9. Relation between the $B-K^{\prime}$ colour and the $K^{\prime}$ magnitude

jects. The scatter in this relation is significantly lower than in the colour - morphological type relation, indicating that the history of star formation, as traced by the $B-K^{\prime}$ colour, is probably more closely related to the mass of the galaxies than to the morphological class. No systematic differences are observed between cluster core and periphery objects. Given the low scatter, many authors (Wyse 1982; Tully et al. 1982; Bothun 1984) suggested to use the NIR colour-magnitude relation as a distance indicator. Figure 9 shows however that the dispersion in the $B-K^{\prime}$ versus $K^{\prime}$ relation is $\sigma_{K^{\prime}} \sim 1.0 \mathrm{mag}$, thus significantly higher than the one obtained with other distance indicators such as the Tully-Fisher relation (Bothun 1984; Jacoby et al. 1992).

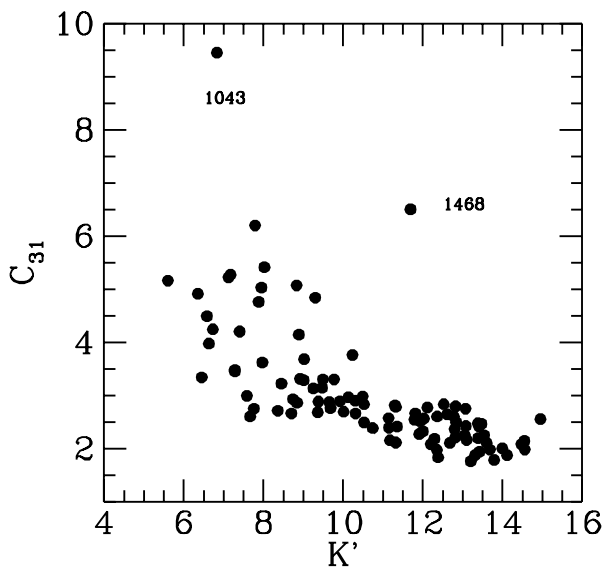

Fig. 10. Relation between the concentration index (at the $22.0 \mathrm{mag} \operatorname{arcsec}^{-2}$ isophote) and the $K^{\prime}$ magnitude

All galaxies with $K^{\prime}>10.0$ mag have a concentration index $\leq 3$, which is consistent with an exponential disc (Gavazzi et al. 1996c), independent of their morphological 
type (see Fig. 10). Stars are more concentrated in bulges and/or in discs only in luminous spirals $\left(K^{\prime} \leq 10.0 \mathrm{mag}\right)$. The presence of bulges and of steep discs in galaxies is thus better discriminated by the NIR luminosity (or by the mass) than by the morphological type. An accurate analysis of the colour-magnitude relation and of the concentration index-magnitude relation extended to a complete sample including dwarf and giant galaxies is addressed in Gavazzi et al. (1996c).

\subsection{The NIR morphology of galaxies}

Inspection of Fig. 3 clearly shows that the majority of the surveyed galaxies can be represented with exponential light profiles, typical of disks. Only a minority - generally bright early type spirals - show the characteristic signature of the bulge. Other features which characterize the morphology in the visible bands are also present in the NIR. Spiral arms, bars and rings are evident in spiral galaxies (see for example VCC 1615) albeit more diffuse and less clumpy. Many of the morphological differences observed between $B$ and $K^{\prime}$ images are due to the more drastic effect of dust obscuration at optical wavelengths. A typical case is the edge-on VCC 92 (NGC 4192), which is one of the largest galaxies in the Virgo cluster. In the $B$ band (Boselli et al., in preparation) a prominent dust lane is observable across the nucleus, and two spiral arms are clearly visible in the outer parts. The $K^{\prime}$ image shows a bright boxy-shaped central region and the two spiral arms close to the centre of the galaxy, not observable in the $B$ band because of dust extinction.

In some spiral galaxies, however, giant HII regions can occasionally be seen in the NIR images. An example is VCC 66 (NGC 4178), where two giant HII regions are observable in the $\mathrm{S}-\mathrm{W}$ part of the disc, about $80-120$ arcsec from the nucleus. At these wavelengths, the emission of the HII regions is dominated by relatively young $\left(10^{7} \mathrm{yr}\right)$ red supergiant stars (Mas-Hesse \& Kunth 1991), while in the $B$ band and in particular in $\mathrm{H} \alpha$ the emission is dominated by young and massive OB stars $\left(10^{6} \mathrm{yr}\right)$ (Kennicutt 1990). Low surface brightness galaxies are faint and diffuse in the NIR. Compact structures in the NIR are more common in BCD galaxies and contribute significantly to the integrated emission. As for giant spirals, these clumpy structures are HII regions, and in the NIR their emission is probably dominated by relatively young red supergiants. On the other hand, HII regions visible in $\mathrm{H} \alpha$ and in $B$ band are sometimes not seen in the NIR; in this case the HII regions are probably too young $\left(\tau \leq 10^{7}\right.$ yr) to have produced red supergiants. The comparison of NIR with optical and $\mathrm{H} \alpha$ images could be a useful tool to evaluate the age of HII regions within galaxies; some preliminary comparisons between $\mathrm{H} \alpha$ and $K^{\prime}$ images are given by Boselli et al. (1995b). We plan to use the available NIR images in conjunction with new $\mathrm{H} \alpha$ (Hippelein et al. 1996) images to study the recent star formation history of these
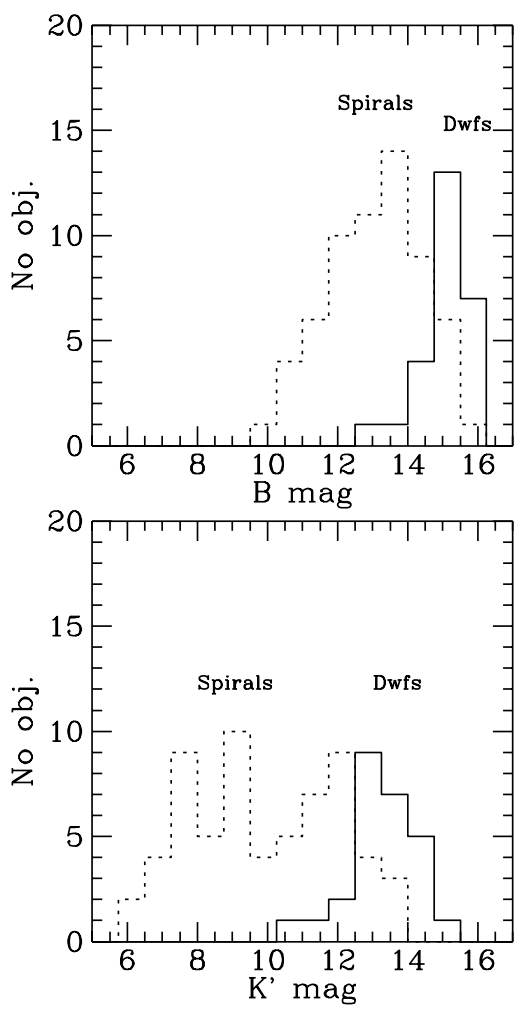

Fig. 11. a) The $B$ luminosity function of the complete volume-limited sample of 88 late-type galaxies $(\geq \mathrm{Sa}$ ) with $B \leq 16.0$ mag observed in this work. The dashed line indicates the 62 spirals $(\mathrm{Sa} \leq$ type $\leq \mathrm{Sm})$, while the full line shows the 26 dwarfs (Im, BCD). b) as for a) but in the $K^{\prime}$ band

objects and to investigate dependencies on morphological type and environment.

\subsection{The NIR luminosity function of spirals}

The subsample of spiral galaxies with available $K^{\prime}$ data constitutes a complete, volume-limited sample suitable for statistical studies. At present all galaxies in the sample with $B \leq 16.0$ mag and of morphological type S0a or later have been imaged (94 objects). In Fig. 11a we compare the $B$ luminosity function of the 62 spiral galaxies (Sa to $\mathrm{Sm}$; dashed line) with the one of the 26 dwarfs ( $\mathrm{Im}, \mathrm{BCD}$, $\mathrm{Im} / \mathrm{BCD}, \mathrm{Sm} / \mathrm{BCD}$; continuum line) observed in this work (using $B$ magnitudes from Binggeli et al. 1985). Given the limiting magnitude of the VCC catalogue of $18.0 B$ mag, the bell-shaped distribution centered at $B=13.0$ mag represents the intrinsic luminosity function of spiral galaxies in Virgo and, as emphasised by Sandage et al. (1985) is not due to an observational cut-off. On the other hand, the distribution of the dwarfs represents the bright tail $(B>13.0)$ of their luminosity function (see Fig. 19 of Sandage et al. 1985); the cut-off at $B=16.0$ mag corresponds to the magnitude limit of the present observations. Figure 11b shows the $K^{\prime}$ luminosity function of spirals and dwarfs (notice however that this is an "hybrid" $K^{\prime}$ 
luminosity function since galaxies were selected in the $B$ band, thus the sample is not magnitude limited in the $K^{\prime}$ band). Two features are worth mentioning: 1) the average $B-K^{\prime}$ of spirals is $\sim 4.0 \mathrm{mag}$, while that of dwarfs is $\sim 2.0$ mag; 2 ) the $K^{\prime}$ band spiral distribution is significantely broader than the corresponding $B$ band distribution. The difference between the two distributions is in accordance with the $B-K^{\prime}$ versus $K^{\prime}$ relation shown in Fig. 9 (see also Gavazzi et al. 1995b). Galaxies at the faint end $\left(11<K^{\prime}<14\right)$ populate the $14<B<16$ bins, thus they have $<B-K^{\prime}>\sim 2.0$, bluer than average.

\section{Summary}

We have obtained NIR surface photometry of a subsample of 102 mainly late-type galaxies in the Virgo cluster complete to $B_{\mathrm{T}}=16.0$ mag. Magnitudes, diameters, concentration indices within the 21.5 and $22.0 \mathrm{mag} \operatorname{arcsec}^{-2}$ isophote and total $H$ and $K^{\prime}$ magnitudes are derived.

The NIR light profiles cover a substantial fraction of the optical diameter and in most cases are consistent with pure disks. Substantial bulges, producing $c_{31}>3.0$, are found among the reddest and most early type objects.

The overall $B$ and $K^{\prime}$ luminosity distributions are consistent with $\left\langle B-K^{\prime}\right\rangle=4$, but there is a trend, more closely linked to galaxian mass than to morphology, for the galaxies to become bluer with progressively fainter $K^{\prime}$ magnitudes.

Acknowledgements. We wish to thank B. Binggeli, R. Lenzen, J. Lequeux, H. Völk and C. Xu for their encouragement and advice throughout the conception and realisation of this work. We thank the MAGIC team at MPI für Astronomie for their skillful operational support and for several helpful discussions about data reduction. We thank the referee, R. Peletier, for valuable comments which helped improving the manuscript. A.B. is supported through the Verbundforschung Astronomie/Astrophysik of DARA under grant \# 50-OR95018.

\section{References}

Aaronson H., Huchra J., Mould J., 1979, ApJ 229, 1

Binggeli B., Sandage A., Tammann G.A., 1985, AJ 90, 1681
Binggeli B., Popescu C.C., Tammann G.A., 1993, A\&AS 98, 275

Boehringer H., Briel U.G., Schwarz R.A., Vogues W., Hartner G., Truemper J., 1994, Nat 368, 828

Boselli A., Gavazzi G., 1994, A\&A 283, 12

Boselli A., Casoli F., Lequeux J., 1995a, A\&AS 110, 521

Boselli A., Gavazzi G., Hippelein H., et al., 1996, in: Minniti D. and Rix H.-W. (eds.) "Spiral Galaxies in the Near-IR". Springer-Verlag, pp. 210-213

Bothun G., 1984, ApJ 277, 532

Elias J.H., Frogel J.A., Matthews K., Neugebauer G., 1982, AJ 87, 1029

Gavazzi G., Scodeggio M., Boselli A., Trinchieri G., 1991, ApJ 382,19

Gavazzi G., 1993, ApJ 419, 469

Gavazzi G., Garilli B., Carrasco L., Boselli A., Cruz Gonzalez I., 1994, A\&AS 104, 271

Gavazzi G., Boselli A., 1996, Astro. Lett. Comm. 35, 1

Gavazzi G., Boselli A., Carrasco L., 1995a, A\&AS 112, 257

Gavazzi G., Randone I., Branchini E., 1995b, ApJ 438, 590

Gavazzi G., Pierini D., Baffa C., 1996a, A\&AS 120, 521

Gavazzi G., Pierini D., Boselli A., Tuffs R., 1996b, A\&AS 120, 489

Gavazzi G., Pierini D., Boselli A., 1996c, A\&A 312, 397

Herbst T., Beckwith S., Birk C., et al., 1993, SPIE 1946

Hippelein H., et al., 1997 (in preparation)

Hoffman L., Helou G., Salpeter E., Lewis M., 1989, ApJ 339, 812

Kennicutt R., 1990, in The Interstellar Medium in Galaxies, Thronson A., Shull J. (eds.). Dordrecht Kluwer, p. 405

Jacoby J., Branch D., Ciardullo R., et al., 1992, PASP 104, 599 Mas-Hesse J., Kunth D., 1991, A\&AS 88, 399

Niklas S., Klein U., Wielebinski R., 1995, A\&A 293, 56

Nilson P., 1973, Uppsala General Catalogue of Galaxies, Uppsala Obs. Ann. Vol. 6 (UGC)

Peletier R.F., Willner S.P., 1991, ApJ 382, 382

Peletier R.F., Willner S.P., 1992, AJ 103, 1761

Pierce M., Tully B., 1988, ApJ 330, 579

Sandage A., Binggeli B., Tammann G.A., 1985, AJ 90, 1759

Tully R., Mould J., Aaronson M., 1982, ApJ 257, 527

Tully R.B., Shaya E.J., 1984, ApJ 281, 31

Wainscoat R., Cowie L., 1992, AJ 103, 332

Whitmore B., 1984, ApJ 278, 61

Wyse R., 1982, MNRAS 199, 1 